\title{
Spatiotemporal structure of biphoton entanglement in type-II parametric down-conversion
}

\author{
E. Brambilla, L. Caspani, L. A. Lugiato, and A. Gatti \\ Istituto Nazionale per la Fisica della Materia-Consiglio Nazionale delle Ricerche-Consorzio Nazionale Interuniversitario per le Scienze \\ Fisiche della Materia, Dipartimento di Fisica e Matematica, Università dell'Insubria, Via Valleggio 11, I-22100 Como, Italy
}

(Received 21 April 2010; published 26 July 2010)

\begin{abstract}
We investigate the spatiotemporal structure of the biphoton correlation in type-II parametric down-conversion (PDC). As in type-I PDC [Phys. Rev. Lett. 102, 223601 (2009)], we find that the correlation is nonfactorizable in space and time. Differently from type I, the type-II correlation in the spontaneous regime displays an asymmetric V-shaped structure in any cross section including time and one transverse dimension. This asymmetry along the temporal coordinate originates from the signal-idler group velocity mismatch and tends to disappear as the parametric gain is raised. We observe a progressive transition toward a symmetric X-shaped geometry similar to that found in type I when stimulated PDC becomes dominant. We also give quantitative evaluations of the localization in space and in time of the correlation, analyze its behavior for different crystal tuning angles, and underline qualitative differences with respect to type-I PDC.
\end{abstract}

DOI: 10.1103/PhysRevA.82.013835 PACS number(s): 42.65.Lm, 42.65.Sf, 42.50.Ar, 42.50.Dv

\section{INTRODUCTION}

Many quantum communication and quantum information schemes exploit the entanglement properties of the photon pairs generated in the process of parametric down-conversion (PDC). In particular, a number of applications in the field of quantum metrology and quantum imaging [1] rely on the strong correlation of the signal and idler photon arrival times and/or transverse positions. Examples are clock synchronization and positioning measurements [2,3], optical coherence tomography [4-6], and nonlinear microscopy [7] where the employment of biphotons with extremely short correlation times is required. Consequently, a number of methods for generating strongly localized biphotons, and more in general to tailor their spectral and temporal properties have been developed in recent years [6,8-11].

In a recent paper [12], we addressed this issue from a novel point of view, by investigating the full spatiotemporal structure of PDC entanglement in the source near field. Considering a type-I phase-matching configuration, we showed that the biphoton amplitude displays a hyperbolic geometry which does not factorize with respect to the space-time coordinates. By looking at cross-section planes along the temporal dimension and one transverse dimension, the structure appears as $\mathrm{X}$-shaped, the $\mathrm{X}$ being traced by the asymptotic behavior of the hyperbola. It can be seen as the microscopic counterpart, at the level of quantum fluctuations, of the "X waves" studied in classical nonlinear optics which form spontaneously in many wave-mixing phenomena [13]. As for these classical objects, the nonfactorizable X-shaped structure arises from the interplay of dispersion and diffraction governed by the phase-matching mechanism, and following this analogy, we coined the name of "X entanglement." More importantly from an applicative point of view, this structure is highly localized both in the spatial (few $\mu \mathrm{m}$ ) and in the temporal (few fs) domain, the correlation time and length of the twin photons being determined by the full spatial and temporal emission bandwidth of the PDC process. The experimental observation of such a geometry would, therefore, allow one to achieve twin photon temporal and spatial localizations in the femtosecond and in the micrometer range. In particular, the predicted temporal localization compares with that obtained in a recent experiment [9], where a 7-fs Hong-Ou-Mandel dip was observed through the use of a chirped quasi-phase-matched nonlinear grating.

In this paper we turn our attention to a type-II phasematching configuration. As is well known, in standard measurement schemes where only small angular portions of the PDC emission are collected, the group velocity mismatch (GVM) between the signal and the idler photons that characterizes type-II crystals drastically deteriorates the correlation of the twin-photon arrival times, a feature which does not occur in type-I PDC. We will show that this issue can be in principle overcome when the full nonfactorizable spatiotemporal geometry of the biphoton amplitude is taken into account. Provided one considers near-field correlation measurements that discriminate the twin photons spatially, one finds indeed an extreme temporal localization of the biphotons in the femtosecond range, similar to what we recently found in type I [12]. However, unlike type-I PDC, the type-II biphotonic structure displays a strong asymmetry along the temporal dimension due to the signal-idler GVM. The temporal region corresponding to "slow" photons arriving before their "fast" twin is indeed forbidden, as long as one considers the purely spontaneous regime of PDC. This makes the biphoton amplitude appear as V-shaped in the allowed half-plane that corresponds to positive time lag of the "slow" photons (like a halved X). It turns out, however, that this asymmetry tends to disappear as the parametric gain is raised and stimulated down-conversion becomes the dominant source of photon pairs: a transition from the $\mathrm{V}$-shaped to an $\mathrm{X}$-shaped structure is observed, recovering thereby the symmetrical structure encountered in type-I phase matching.

The paper is organized as follows: in Secs. II and III we define the spatiotemporal biphoton amplitude $\psi$ and introduce the model used to evaluate $\psi$ in the plane-wave pump limit. In Sec. IV A we describe the approximations that are used in the numerics to evaluate $\psi$ within the paraxial approximation, further details being given in the appendixes. The behavior of the biphoton amplitude is illustrated in Secs. V and VI 
in the coincidences regime and for increasing parametric gains, respectively. In Sec. VII the effect of different phasematching conditions are investigated by considering the crystal orientations.

\section{THE BIPHOTON CORRELATION IN TYPE-II PDC}

We assume a coherent pump field propagating along the $z$-axis direction is injected into a $\chi^{(2)}$ crystal slab cut for type-II phase matching. The signal and idler fields emitted in the PDC process are measured in the near field of the source and we indicate with $B_{1}(\vec{x}, t)$ and $B_{2}(\vec{x}, t)$ the corresponding field operators in the planes where the measurement is performed. Their normalization is such that the expectation value of $N_{j}(\vec{x}, t)=B_{j}^{\dagger}(\vec{x}, t) B_{j}(\vec{x}, t)$ gives the mean photon flux density of field $j$ as a function of time and the transverse spatial coordinates $\vec{x}=(x, y)$. The quantity of interest is the spatiotemporal cross-correlation function of the signal and idler intensities:

$$
\begin{gathered}
G^{(2)}\left(\vec{x}_{1}, t_{1}, \vec{x}_{2}, t_{2}\right)=\left\langle N_{1}\left(\vec{x}_{1}, t_{1}\right) N_{2}\left(\vec{x}_{2}, t_{2}\right)\right\rangle \\
=\left\langle N_{1}\left(\vec{x}_{1}, t_{1}\right)\right\rangle\left\langle N_{2}\left(\vec{x}_{2}, t_{2}\right)\right\rangle+\left|\psi\left(\vec{x}_{1}, t_{1}, \vec{x}_{2}, t_{2}\right)\right|^{2} .
\end{gathered}
$$

The last equality derives from the Gaussian factorization properties of the fourth-order PDC correlation function which can be expressed as a sum of products of secondorder field correlation functions together with the relation $\left\langle B_{1}^{\dagger}\left(\vec{x}_{1}, t_{1}\right) B_{2}\left(\vec{x}_{1}, t_{1}\right)\right\rangle=0$ that holds for type-II phase matching. The first term in Eq. (1b), the product of the measured photon number, usually acts as a background term, while the signal-idler field correlation function, also called biphoton amplitude,

$$
\psi\left(\vec{x}_{1}, t_{1}, \vec{x}_{2}, t_{2}\right)=\left\langle B_{1}\left(\vec{x}_{1}, t_{1}\right) B_{2}\left(\vec{x}_{2}, t_{2}\right)\right\rangle,
$$

contains all the desired information about the spatiotemporal structure of the PDC correlation. We shall see in the next section that in the regime of low parametric gain $|\psi|^{2}$ scales as the number of photons $\left\langle N_{1}\right\rangle$, so that when the number of detected photons is small the correlation term prevails with respect to the background term $\left\langle N_{1}\right\rangle\left\langle N_{2}\right\rangle$ in Eq. (1b). At low parametric gain $G^{(2)} \approx|\psi|^{2}$ reproduces, therefore, the measured coincidence rate as a function of the spatial and temporal coordinates of the emitted twin photons: $\left|\psi\left(\vec{x}_{1}, t_{1}, \vec{x}_{2}, t_{2}\right)\right|^{2}$ gives the joint probability of detecting a signal photon in position $\vec{x}_{1}$ at time $t_{1}$ and an idler photon in position $\vec{x}_{2}$ at time $t_{2}$. In this same regime, the biphoton amplitude can be analytically evaluated in the Fourier domain in the general case where the pump beam has a finite transverse size and duration [12,14,15]. On the contrary, in a regime of high parametric gain such an analytical expression does not exist, except in the limit of a stationary monochromatic plane-wave pump (PWP). In [12,14] we have shown that the biphoton amplitude in direct space (space and time) is well approximated by the PWP result provided the duration and waist of the pump pulse are sufficiently large compared to the temporal and spatial walk-off between the ordinary and extraordinary waves. For our purpose, we shall assume this condition of quasistationarity is fulfilled, so that $\psi$ can be evaluated at any gain regime from the PWP theory as a function of the relative spatial $\left(\vec{x}_{1}-\vec{x}_{2}\right)$ and temporal coordinates $\left(t_{1}-t_{2}\right)$. This will allow us to illustrate straightforwardly the transition from the low-gain to the high-gain regime.

\section{MODELING BROADBAND PDC IN THE PLANE-WAVE PUMP LIMIT}

In this section we illustrate the model we use to investigate the PDC quantum correlation in the PWP limit. It is similar to that described in $[16,17]$, except we shall not perform the usual quadratic dispersion approximation with the purpose of describing the full bandwidth of the PDC emission process in the temporal frequency domain.

In the regime where pump depletion effects are assumed negligible, the pump field can be treated as a coherent $c$-number field that propagates linearly. We shall assume it is a monochromatic plane wave of frequency $\omega_{0}$ and wave number $k_{0}=\omega_{0} n_{0} / c$ that propagates along the $z$-axis direction. The signal and idler fields are treated as quantized multimode fields. We denote with $A_{1}(z, \vec{x}, t)$ and $A_{2}(z, \vec{x}, t)$ their respective envelope operators, taken with respect to the common central frequencies $\omega_{s} \equiv \omega_{1}=\omega_{2}=\omega_{0} / 2$ and wave numbers $k_{0} / 2$. We introduce their Fourier transforms with respect to time and to the transverse spatial coordinates $\vec{x}=(x, y)$,

$A_{j}(z, \vec{q}, \Omega)=\int \frac{d^{2} \vec{x}}{2 \pi} \int \frac{d t}{\sqrt{2 \pi}} A_{j}(z, \vec{x}, t) e^{-i \vec{q} \cdot \vec{x}+i \Omega t}, \quad(j=1,2)$,

where $\Omega$ is the frequency offset with respect to the degenerate frequency $\omega_{s}=\omega_{0} / 2$ and $\vec{q}$ is the transverse wave-vector component. The propagation equation for the signal field $A_{1}$ in Fourier space takes then the simple form,

$$
\begin{aligned}
\frac{\partial A_{1}(z, \vec{q}, \Omega)}{\partial z}= & i\left[k_{1 z}(\vec{q}, \Omega)-\frac{k_{0}}{2}\right] A_{1}(z, \vec{q}, \Omega) \\
& +\frac{g}{l_{c}} A_{2}^{\dagger}(z,-\vec{q},-\Omega) .
\end{aligned}
$$

The coupled equation for the idler field $A_{2}$ is obtained by exchanging the wave indexes $1 \leftrightarrow 2$. The first term on the right-hand side describes the linear propagation of field $j$, with $k_{j z}(\vec{q}, \Omega)=\sqrt{k_{j}^{2}(\vec{q}, \Omega)-q^{2}}$ indicating the $z$ component of the $k$ vector associated to the $(\vec{q}, \Omega)$ Fourier mode. Rather than performing the usual expansion in power of $\Omega$, in the numerics (see Secs.V and VI), we shall evaluate the wave numbers,

$$
k_{j}(\vec{q}, \Omega)=\frac{\omega_{s}+\Omega}{c} n_{j}(\vec{q}, \Omega),
$$

by using the complete Sellmeier dispersion formula giving the refraction indexes $n_{j}(\vec{q}, \Omega)$ for a broad range of temporal frequencies [18]. This is required by our interest in modeling the full bandwidth of the PDC emission. Notice also that the case of anisotropic propagation is here taken into account through the assumption of an explicit dependence of $n_{j}(\vec{q}, \Omega)$ on the transverse wave vector $\vec{q}$. For the $\beta$ barium-borate (BBO) crystal cut for type-II phase matching we shall consider for the numerical examples, this occurs for the pump field and one of the two down-converted fields, 
say the idler field $j=2$, which are both extraordinarily polarized.

The second term in Eq. (4) describes the nonlinear interaction of the signal and idler waves. Its strength is determined by the parametric gain $g$, a dimensionless quantity proportional to the pump field peak value, to the crystal length $l_{c}$ and to the effective second-order nonlinearity of the $\chi^{(2)}$ medium. By solving Eq. (4), the field operators at the crystal output face $B_{j}(\vec{q}, \Omega) \equiv A_{j}\left(z=l_{c}, \vec{q}, \Omega\right)$ can be expressed as functions of those at the entrance face $A_{j}(\vec{q}, \Omega) \equiv A_{j}(z=0, \vec{q}, \Omega)$ through a unitary input-output transformation of the form $[16,17]$,

$$
\begin{aligned}
& B_{1}(\vec{q}, \Omega)=U_{1}(\vec{q}, \Omega) A_{1}(\vec{q}, \Omega)+V_{1}(\vec{q}, \Omega) A_{2}^{\dagger}(-\vec{q},-\Omega), \\
& B_{2}(\vec{q}, \Omega)=U_{2}(\vec{q}, \Omega) A_{2}(\vec{q}, \Omega)+V_{2}(\vec{q}, \Omega) A_{1}^{\dagger}(-\vec{q},-\Omega) .
\end{aligned}
$$

We see that only conjugate modes of opposite transverse wave vector $\pm \vec{q}$ and conjugate frequencies $\omega_{0} / 2 \pm \Omega$ are coupled, a feature expressing the perfect entanglement of the twin-photon energy and transverse momentum taking place in the PWP limit. The explicit expression of the gain function $U_{j}, V_{j}$ can be found in $[16,17]$. We mention here that they satisfy the unitarity conditions,

$$
\begin{gathered}
\left|U_{j}(\vec{q}, \Omega)\right|^{2}-\left|V_{j}(\vec{q}, \Omega)\right|^{2}=1, \quad(j=1,2), \\
U_{1}(\vec{q}, \Omega) V_{2}(-\vec{q},-\Omega)=U_{2}(-\vec{q},-\Omega) V_{1}(\vec{q}, \Omega),
\end{gathered}
$$

which guarantee the preservation of the commutation relations at equal $z$ :

$$
\begin{gathered}
{\left[A_{i}(z, \vec{q}, \Omega), A_{j}^{\dagger}\left(z, \vec{q}^{\prime}, \Omega^{\prime}\right)\right]=\delta_{i j} \delta\left(\vec{q}-\vec{q}^{\prime}\right) \delta\left(\Omega-\Omega^{\prime}\right),} \\
{\left[A_{i}(z, \vec{q}, \Omega), A_{j}\left(z, \vec{q}^{\prime}, \Omega^{\prime}\right)\right]=0, \quad(i, j=1,2) .}
\end{gathered}
$$

Using these commutation rules together with the fact that the input fields are in the vacuum state, the PDC field correlation function in Fourier space can be readily evaluated, giving $[16,17]$

$$
\begin{aligned}
\left\langle B_{i}^{\dagger}(\vec{q}, \Omega) B_{j}\left(\vec{q}^{\prime}, \Omega^{\prime}\right)\right\rangle= & \delta_{i, j} \delta\left(\vec{q}-\vec{q}^{\prime}\right) \delta\left(\Omega-\Omega^{\prime}\right) \\
& \times\left|V_{j}(\vec{q}, \Omega)\right|^{2}, \\
\left\langle B_{i}(\vec{q}, \Omega) B_{j}\left(\vec{q}^{\prime}, \Omega^{\prime}\right)\right\rangle= & \left(1-\delta_{i, j}\right) \delta\left(\vec{q}+\vec{q}^{\prime}\right) \delta\left(\Omega+\Omega^{\prime}\right) \\
& \times U_{i}(\vec{q}, \Omega) V_{j}(-\vec{q},-\Omega) \quad(i, j=1,2) .
\end{aligned}
$$

The last relations can be used in order to evaluate the near-field biphoton amplitude (2). Noticing that the stationary and homogeneous conditions that characterize the PWP limit lead to a dependence on the spatiotemporal coordinates only through the relative position $\vec{x}=\vec{x}_{1}-\vec{x}_{2}$ and time delay $t=t_{1}-t_{2}$, we find

$$
\begin{aligned}
\psi & \left(\vec{x}_{1}-\vec{x}_{2}, t_{1}-t_{2}\right) \\
& =\int \frac{d \vec{q}}{(2 \pi)^{2}} \int \frac{d \Omega}{2 \pi} e^{i \vec{q} \cdot\left(\vec{x}_{1}-\vec{x}_{2}\right)-i \Omega\left(t_{1}-t_{2}\right)} U_{1}(\vec{q}, \Omega) V_{2}(-\vec{q},-\Omega) .
\end{aligned}
$$

We need, therefore, to evaluate the Fourier transform of the function $U_{1}(\vec{q}, \Omega) V_{2}(-\vec{q},-\Omega)$, whose explicit expression reads

$$
\begin{aligned}
F_{12}(\vec{q}, \Omega) \equiv & U_{1}(\vec{q}, \Omega) V_{2}(-\vec{q},-\Omega)=\frac{g \sinh \Gamma(\vec{q}, \Omega)}{\Gamma(\vec{q}, \Omega)} \\
& \times\left[\cosh \Gamma(\vec{q}, \Omega)+i \frac{\Delta(\vec{q}, \Omega) l_{c}}{2 \Gamma(\vec{q}, \Omega)} \sinh \Gamma(\vec{q}, \Omega)\right] \\
& \Gamma(\vec{q}, \Omega)=\sqrt{g^{2}-\frac{\Delta^{2}(\vec{q}, \Omega) l_{c}}{4}}
\end{aligned}
$$

where

$$
\Delta(\vec{q}, \Omega)=\left[k_{1 z}(\vec{q}, \Omega)+k_{2 z}(-\vec{q},-\Omega)-k_{0}\right]
$$

is the PWP phase-mismatch function, which depends on the dispersion properties in the nonlinear medium of both the ordinary and the extraordinary waves. Notice that generally $\psi(\vec{x}, t)$ is not even with respect to the space-time coordinates, as a consequence the spatiotemporal walk-off between the ordinary and the extraordinary waves. This feature characterizes type II with respect to type-I PDC, as in the latter case the signal and idler waves are identically polarized [i.e., $k_{1}(\vec{q}, \Omega) \equiv k_{2}(\vec{q}, \Omega)$ ] and both the phase-mismatch function (12) and the biphoton amplitude (10) are symmetric functions of their arguments.

Other quantities of interest are the near-field self-correlation functions $\left\langle B_{j}^{\dagger}(\vec{x}, t) B_{j}\left(\vec{x}^{\prime}, t^{\prime}\right)\right\rangle$, which describe the phasecoherence properties of each down-converted field in the space-time domain. They can be written in the form,

$$
\begin{aligned}
& \left\langle B_{j}^{\dagger}(\vec{x}, t) B_{j}\left(\vec{x}^{\prime}, t^{\prime}\right)\right\rangle \\
& =\int \frac{d \vec{q}}{(2 \pi)^{2}} \int \frac{d t}{2 \pi} e^{-i \vec{q} \cdot\left(\vec{x}-\vec{x}^{\prime}\right)+i \Omega\left(t-t^{\prime}\right)}\left|V_{j}(\vec{q}, \Omega)\right|^{2}, \quad(j=1,2),
\end{aligned}
$$

as it can be inferred by using Eq. (9b). This relation expresses a generalization of the classical Wiener-Khintchine theorem [19] extended to the space-time domain, since the functions,

$$
\begin{gathered}
F_{11}(\vec{q}, \Omega) \equiv\left|V_{1}(\vec{q}, \Omega)\right|^{2}=g^{2} \frac{\sinh ^{2} \Gamma^{2}(\vec{q}, \Omega)}{\Gamma^{2}(\vec{q}, \Omega)}, \\
F_{22}(\vec{q}, \Omega) \equiv\left|V_{2}(\vec{q}, \Omega)\right|^{2}=\left|V_{1}(-\vec{q},-\Omega)\right|^{2},
\end{gathered}
$$

are the photon number density fluxes of the signal and idler fields in Fourier space and can be identified with their spatiotemporal emission spectra. By exploiting this relationship, the spatiotemporal structure of PDC coherence has been recently investigated experimentally through angularly resolved spectral measurements in the case of a type-I BBO crystal in a regime of high parametric gain $[20,21]$.

The functions $F_{11}$ and $F_{22}$ depend on the Fourier coordinates only through the phase-mismatch functions $\Delta( \pm \vec{q}, \pm \Omega) l_{c}$ (the plus sign holds here for $F_{11}$, the minus sign for $\left.F_{22}\right)$ and we have $F_{11}(\vec{q}, \Omega)=F_{22}(-\vec{q},-\Omega)$ according to unitarity conditions (7). They are strongly peaked in Fourier space regions where $\Delta( \pm \vec{q}, \pm \Omega) l_{c}=0$, as efficient downconversion occurs only for those conjugate signal-idler mode pairs, $(\vec{q}, \Omega)$ and $(-\vec{q},-\Omega)$, for which the accumulated phase mismatch is small. 
In the low-gain limit (i.e., for $g \ll 1$ ), $F_{12}$ and $F_{11}$ are well approximated by the expressions,

$$
\begin{gathered}
F_{12}(\vec{q}, \Omega) \approx V_{1}(\vec{q}, \Omega) \approx g e^{i \frac{\Delta(\vec{q}, \Omega) l_{c}}{2}} \operatorname{sinc}\left[\frac{\Delta(\vec{q}, \Omega) l_{c}}{2}\right], \\
F_{11}(\vec{q}, \Omega) \equiv\left|V_{1}(\vec{q}, \Omega)\right|^{2} \approx g^{2} \operatorname{sinc}^{2}\left[\frac{\Delta(\vec{q}, \Omega) l_{c}}{2}\right],
\end{gathered}
$$

where $\operatorname{sinc}(x) \equiv \sin (x) / x$. We see from this result and Eqs. (10) and (11) that both the mean photon fluxes $\left\langle N_{j}\right\rangle=$ $\int \frac{d \vec{q}}{2 \pi} \int \frac{d \Omega}{\sqrt{2 \pi}}\left|V_{j}(\vec{q}, \Omega)\right|^{2}$ and the square modulus of the biphoton amplitude $|\psi|^{2}$ scales as $g^{2}$ for $g \rightarrow 0$. Thus, as anticipated in Sec. II, the first term in Eq. (1b), namely the background term $\left\langle N_{1}\right\rangle\left\langle N_{2}\right\rangle$, becomes negligible with respect to the correlation term $|\psi|^{2}$ at low parametric gains.

\section{APPROXIMATIONS FOR $\Delta(\vec{q}, \Omega) l_{c}$}

In this section we illustrate the different levels of approximation for the phase-mismatch function that are used in the numerical and analytical calculations. From now on we shall refer to the case of a uniaxial crystal with $e$-oe phase matching. In particular, we shall take as an example the case of a BBO crystal cut for type II with an extraordinarily polarized plane-wave pump field injected at $\lambda_{0}=352 \mathrm{~nm}$, with the down-converted ordinary $(j=1)$ and extraordinary $(j=2)$ fields being referred to as the signal and idler wave, respectively.

\section{A. Paraxial approximation: reduction to the Hankel transform}

The first degree of approximation we shall consider is the paraxial approximation used in the numerics. It is obtained by expanding $\Delta(\vec{q}, \Omega) l_{c}$ in powers of $q_{x}$ and $q_{y}$ up to quadratic terms, while keeping the dependence on $\Omega$ expressed by the Sellmeier relation. This approximation allows one to reduce the two-dimensional (2D) Fourier transform in the spatial frequency space that defines the biphoton amplitude (10) to a one-dimensional Hankel transform. This feature substantially reduces the time of computation required to evaluate $\psi(\vec{x}, t)$ numerically, though maintaining a good precision level. We find that the phase-mismatch function can be written in the form (see Appendix A for a detailed derivation),

$$
\Delta(\bar{q}, \Omega) l_{c} \approx D_{0}(\Omega)-\frac{\bar{q}^{2}}{q_{0}^{2}(\Omega)},
$$

where $\bar{q}=\sqrt{\bar{q}_{x}^{2}+\bar{q}_{y}^{2}}$ denotes the radial coordinate of the scaled spatial frequencies,

$$
\begin{gathered}
\bar{q}_{x}=\beta_{x}\left[q_{x}-q_{C}(\Omega)\right], \\
\bar{q}_{y}=\beta_{y} q_{y} .
\end{gathered}
$$

The parameters depending on $\Omega$ in Eqs. (16) and (17a) are given by

$$
\begin{gathered}
q_{0}(\Omega)=\sqrt{\frac{\bar{k}(\Omega)}{l_{c}},} \\
\bar{k}(\Omega)=\frac{2 k_{1}(\Omega) k_{2}(\vec{q}=0,-\Omega)}{k_{1}(\Omega)+k_{2}(\vec{q}=0,-\Omega)},
\end{gathered}
$$

$$
\begin{gathered}
q_{C}(\Omega)=\frac{1}{2 \beta_{x}^{2}} \bar{k}(\Omega) \rho_{2}(-\Omega), \\
D_{0}(\Omega)=\left[k_{1}(\Omega)+k_{2}(\vec{q}=0,-\Omega)-k_{0}\right] l_{c} \\
+\frac{1}{4 \beta_{x}^{2}} \bar{k}(\Omega) l_{c} \rho_{2}^{2}(-\Omega),
\end{gathered}
$$

with $\rho_{2}(\Omega)=-\left(\partial k_{2} / \partial q_{x}\right)_{\vec{q}=0}$ denoting the walk-off angle of the idler wave with respect to the pump axis direction. Its relation to the pump direction angle $\theta_{0}$ (with respect to the crystal axis) and the ordinary and extraordinary principal axis refraction indexes, $n_{o}(\Omega)$ and $n_{e}(\Omega)$, is given in Eq. (A5) of Appendix A. Note that according to its definition and the chosen reference frame orientation illustrated in Fig. 11 of the appendix, both $\rho_{2}$ and $q_{C}$ are positive for the negative BBO crystal. The dimensionless coefficients $\beta_{x}$ and $\beta_{y}$ are defined through the relations,

$$
\begin{gathered}
\beta_{x}^{2}=1-\frac{k_{1}}{k_{1}+k_{2}}\left(\rho_{2}^{2}-\frac{2 \rho_{2}}{\tan 2 \theta_{0}}\right), \\
\beta_{y}^{2}=1+\frac{k_{1}}{k_{1}+k_{2}} \frac{\rho_{2}}{\tan \theta_{0}},
\end{gathered}
$$

where we used the short-hand notation $\rho_{2} \equiv \rho_{2}(\Omega=0), k_{j} \equiv$ $k_{j}(\vec{q}=0, \Omega=0)$. We chose to ignore the slow dependence on $\Omega$ of the parameters $\beta_{x}, \beta_{y}$, after checking that this does not affect substantially the precision of the approximation. The phase-matching "curve," that is, the surface in the $\left(q_{x}, q_{y}, \Omega\right)$ space defined by the equation,

$$
\Delta(\vec{q}, \Omega) l_{c}=0,
$$

exists only in the frequency region where $D_{0}(\Omega) \geqslant 0$. In such a region, a section at a fixed $\Omega$ is an ellipse in the $\left(q_{x}, q_{y}\right)$ plane (i.e., in the far-field plane) centered on $\left(q_{x}=q_{C}(\Omega), q_{y}=0\right)$ with the semiaxes $q_{0}(\Omega) \sqrt{D_{0}(\Omega)} / \beta_{x}$ and $q_{0}(\Omega) \sqrt{D_{0}(\Omega)} / \beta_{y}$ parallel to the $q_{x}$ and $q_{y}$ axes. However, their eccentricity is usually rather small, since both $\beta_{x}$ and $\beta_{y}$ are close to unity. In the type-II BBO case considered in the numerics, we find $\beta_{x}^{2}=0.9871<\beta_{y}^{2}=1.0316$ for $\theta_{0}=49.05^{\circ}$; the ellipse is, therefore, slightly elongated along the $q_{x}$ axis (parallel to the walk-off plane) with the small eccentricity $\sqrt{1-\beta_{x}^{2} / \beta_{y}^{2}}=$ 0.208 and can be hardly distinguished from a circumference of radius $q_{0}(\Omega) \sqrt{D_{0}(\Omega)}$. Expression (16) provides an excellent approximation for the phase-mismatch function (12) over a broad range of temporal frequencies. This is shown in Fig. 1 where the phase-matching curve $\Delta(\vec{q}, \Omega) l_{c}=0$ in the $\left(\Omega, q_{x}\right)$ plane for $q_{y}=0$ obtained from Eq. (16) (solid line) is compared with that obtained numerically without any approximation using an iterative algorithm (hollow circle).

Exploiting the radial symmetry of the phase-mismatch function with respect to the scaled spatial frequencies (17), Eq. (10) can be rewritten as a Hankel transform,

$$
\begin{aligned}
\psi(\overline{\bar{x}}, t)= & \frac{1}{\beta_{x} \beta_{y}} \int \frac{d \Omega}{2 \pi} e^{-i \Omega t} e^{i q_{C}(\Omega) \bar{x}}, \\
& \int_{0}^{\infty} \frac{d \bar{q}}{2 \pi} \bar{q} J_{0}(\bar{q} \bar{r}) F_{12}(\bar{q}, \Omega),
\end{aligned}
$$




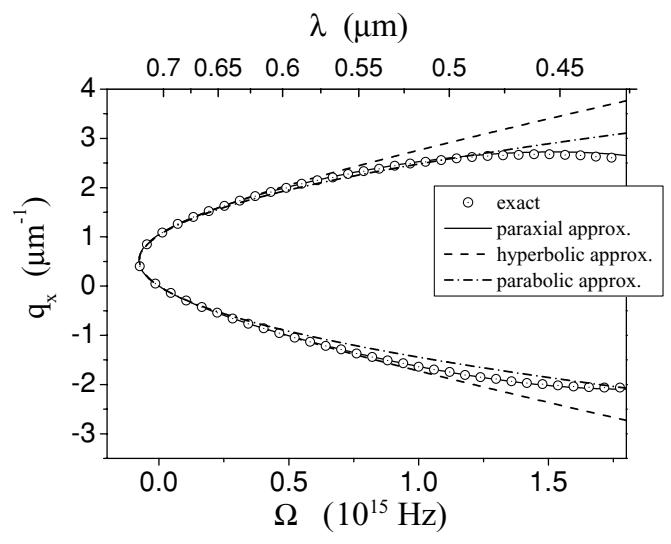

FIG. 1. The phase-matching curve in the plane $\left(q_{x}, \Omega\right)$ as obtained from Eq. (16) (solid line) is compared with the numerical result without paraxial approximation (hollow circle). The dashed line and the dotted-dashed line correspond, respectively, to the hyperbolic and the parabolic approximations expressed by Eqs. (26) and (29). In this example the pump axis direction $\left(\theta_{0}=49.05^{\circ}\right)$ is such that collinear phase matching occurs at degeneracy (i.e., $\Delta_{0} l_{c}=0$ ).

where $J_{0}(x)=\frac{1}{2 \pi} \int_{0}^{2 \pi} e^{i x \sin (\theta)} d \theta$ is the $0^{\text {th }}$ order Bessel function and

$$
\bar{x}=x / \beta_{x}, \quad \bar{y}=y / \beta_{y}, \quad \bar{r}=\sqrt{\bar{x}^{2}+\bar{y}^{2}}
$$

are the scaled spatial coordinates. In the numerics, we first evaluated the Hankel transform in Eq. (21) by using the quadrature method illustrated in [22] which offers an excellent precision level. The Fourier transform with respect to the temporal frequency was evaluated using the FFT-based Bluestein algorithm which allows one to select an appropriate time window within the Nyquist interval without resorting to zero padding [23]. Especially at low gain, the slow decaying and strongly oscillating behavior of $F_{12}$ requires a large number of quadrature points, so that the direct evaluation of the (2+1) $D$ transform given in Eq. (10) would have been much more demanding, if not prohibitive, from the point of view of both computation times and memory use.

\section{B. Quadratic (hyperbolic) approximation in $\vec{q}$ and $\Omega$}

As can be seen from Fig. 1, the approximation illustrated in Sec. IV B, Eq. (16) provides an excellent fit with the exact phase-matching curve. In order to obtain analytical results, we shall also resort to a cruder approximation holding close to degeneracy, obtained by expanding $D_{0}(\Omega)$ quadratically and setting $\beta_{x}=\beta_{y}=1$ in Eqs. (16) and (18d) [this corresponds to neglecting the second-order derivatives of $k_{2}(\vec{q}, \Omega)$ with respect to $q_{x}, q_{y}$ given in Appendix A, Eq. (A6)]:

$$
\Delta(\bar{q}, \Omega) l_{c}=D_{0}+\frac{\Omega}{\Omega_{0}}+\frac{\Omega^{2}}{\Omega_{0}^{\prime 2}}-\frac{\bar{q}^{2}}{q_{0}^{2}} .
$$

In this expression the parameters $q_{0}=\sqrt{\bar{k} / l_{c}}, \quad \bar{k}=$ $2 k_{1} k_{2} /\left(k_{1}+k_{2}\right), q_{C}=\frac{1}{2} \bar{k} \rho_{2}$, and $D_{0}=\Delta_{0} l_{c}+\frac{1}{4} \bar{k} l_{c} \rho_{2}^{2}$ are the constants obtained by setting $\beta_{x}=\beta_{y}=1$ and $\Omega=0$ into Eqs. (18a)-(18d), with

$$
\Delta_{0}=k_{1}+k_{2}-k_{0}
$$

denoting the collinear phase-mismatch parameter at degeneracy. Notice that if $D_{0}>0$, the quantity $q_{R}=q_{0} \sqrt{D_{0}}$ gives the radius of the phase-matching circumference in the $\left(q_{x}, q_{y}\right)$ plane at degeneracy. Using the short-hand notation $k_{j}^{\prime}=\left(\partial k_{j} / \partial \Omega\right)_{0,0}, k_{j}^{\prime \prime}=\left(\partial^{2} k_{j} / \partial \Omega^{2}\right)_{0,0}$, to denote the $k$-vector frequency derivatives at degeneracy along the pump axis direction (i.e., for $\vec{q}=0, \Omega=0$ ), the other parameters $\Omega_{0}$ and $\Omega_{0}^{\prime \prime}$ appearing in Eq. (23) are defined through the relations,

$$
\Omega_{0}^{-1} \equiv\left(k_{1}^{\prime}-k_{2}^{\prime}\right) l_{c}, \quad \Omega_{0}^{\prime \prime-2} \equiv \frac{1}{2}\left(k_{1}^{\prime \prime}+k_{2}^{\prime \prime}\right) l_{c},
$$

and give the characteristic temporal bandwidths associated to group velocity mismatch (GVM) and group velocity dispersion (GVD), respectively. In type-II phase matching, the walkoff time $t_{\text {coh }}=\Omega_{0}^{-1}$ determines the typical coherence time characterizing the PDC field in a far-field detection scheme, while in type-I phase matching this role is taken by $\Omega_{0}^{\prime \prime-1}$ as in this case the GVM is vanishing at degeneracy ( since $k_{1}^{\prime}=k_{2}^{\prime}$ ).

Taking the roots of expression (23) with respect to $\Omega$, we obtain the two hyperbolic branch in the $(\bar{q}, \Omega)$ plane,

$$
\Omega_{ \pm}(\bar{q})=-\frac{\Omega_{0}}{2 \epsilon} \pm \frac{\Omega_{0}}{2 \epsilon} \sqrt{1+4 \epsilon\left(\frac{\bar{q}^{2}}{q_{0}^{2}}-D_{0}\right)},
$$

where $\epsilon \equiv \Omega_{0}^{2} / \Omega_{0}^{\prime 2}$. For the 4-mm-long BBO crystal considered in the numerical examples (see next sections) we have $\Omega_{0}^{\prime \prime-1}=18.5 \mathrm{fs}, \Omega_{0}^{-1}=985 \mathrm{fs}, \epsilon=3.3 \times 10^{-4}$. Because of the smallness of such parameters, it can be easily verified that only the solution $\Omega_{+}(\bar{q})$ corresponds to a positive frequency $\frac{\omega_{0}}{2}+\Omega>0$, while $\Omega_{-}(\bar{q})$ may be discarded. The plot of $\Omega_{+}(\bar{q})$ (dashed line in Fig. 1) fits well the "exact" phase-matching curve obtained with the use of the complete Sellmeier relation close to degeneracy, but fails for large values of $\Omega$, as expected. The asymptotic limit of this hyperbolic branch for large spatial frequencies (corresponding to high temporal frequencies) is given by

$$
\Omega_{+}(\bar{q}) \approx-\frac{\Omega_{0}}{2 \epsilon}+\frac{\Omega_{0}^{\prime \prime}}{q_{0}} \bar{q}, \text { for } \frac{\bar{q}}{q_{0}} \gg \sqrt{\frac{1}{4 \epsilon}-D_{0}} .
$$

As we shall see in the following section, it turns out that the large-scale geometry of the biphotonic structure in the space-time domain is actually governed by this asymptotic behavior.

\section{Linearization with respect to $\Omega$ (parabolic approximation)}

Some analytical results will be obtained by using an even cruder approximation, which neglects the effect of GVD with respect to GVM, so that the phase-mismatch function (23) becomes linear in $\Omega$,

$$
\Delta_{L}(\bar{q}, \Omega) l_{c}=D_{0}+\frac{\Omega}{\Omega_{0}}-\frac{\bar{q}^{2}}{q_{0}^{2}} .
$$

In the same limit, the hyperbolic phase-matching curve (26) reduces to the parabola

$$
\Omega^{(L)}(\bar{q})=\Omega_{0}\left(\frac{\bar{q}^{2}}{q_{0}^{2}}-D_{0}\right),
$$

which corresponds to the limit of $\Omega_{+}(\bar{q})$ for $\epsilon \rightarrow 0$. For finite values of the parameter $\epsilon$, Eqs. (28) and (29) are good 
approximations of Eqs. (23) and (26) close to degeneracy, in a range of frequencies such that $\left|\Omega / \Omega_{0}\right| \ll 1 / \epsilon$. The plot of (29) is displayed by the dotted-dashed line in Fig. 1.

\section{BEHAVIOR OF THE BIPHOTON AMPLITUDE AT LOW GAINS}

We first consider a regime of low parametric gain, taking $g=0.001$, and considering the same BBO parameters as in Fig. 1: the pump direction, $\theta_{0}=49.05^{\circ}$, is such that the collinear phase matching takes place at the degenerate wavelength $\lambda_{s}=704 \mathrm{~nm}$ (i.e., $\Delta_{0} l_{c}=0$ ).

Figure 2(a) plots on a large scale the phase-matching curves of the signal field (on the right) and of the idler field (on the left) in the plane corresponding to the walk-off direction. In the numerical simulation, both fields were multiplied by a super-Gaussian filter centered at degeneracy with a full width at half-maximum $(\mathrm{FWHM})$ frequency bandwidth $\Delta \Omega_{\mathrm{F}} \approx 1.5$. $10^{15} \mathrm{~Hz}$ corresponding to a $\sim 400$-nm range of wavelength (it is indicated by the unshaded region in Fig. 2). Its purpose is to select an appropriate bandwidth of temporal frequencies that represents, for example, the detection bandwidth.

Figure 3 displays the numerical result for the modulus of the biphoton amplitude in the $(x, t)$ plane on a femtosecond-micrometer scale. We have a kind of V-shaped structure that clearly does not factorize with respect to the space-time coordinates and that extends mainly in the positive $t$ half-plane while it is almost vanishing for $t<0$. This last feature distinguishes type-II phase matching with respect to type-I PDC: the latter displays indeed a perfectly symmetric structure at any gain, both with respect to the spatial and the temporal coordinates and appears as X-shaped [12,14]. The asymmetry of the biphoton amplitude along the temporal dimension displayed in Fig. 3 has a simple physical explanation related to the temporal walk-off between the signal and idler waves that characterizes type-II PDC. As $|\psi(\vec{x}, t)|^{2}$ is proportional to the probability of finding the signal photon in the relative position $\vec{x}=\vec{x}_{1}-\vec{x}_{2}$ and with a time delay

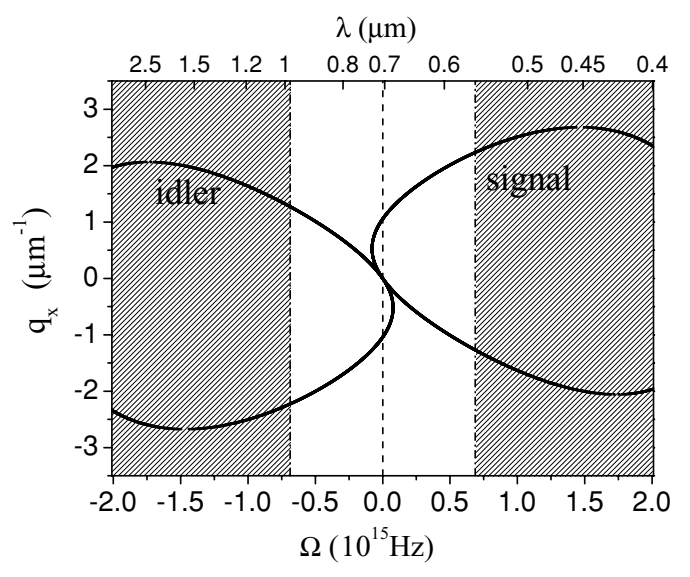

FIG. 2. Plot of the phase-matching curves of the signal and idler waves in the walk-off direction for the same parameters as in Fig. 1. The unshaded region centered at degeneracy corresponds to the chosen frequency range selected by the super-Gaussian filter.
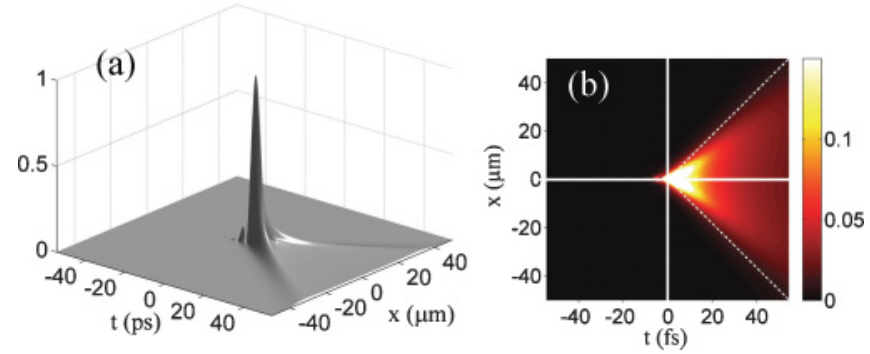

FIG. 3. (Color online) Structure of the modulus of the biphoton amplitude on a fs $/ \mu \mathrm{m}$ scale as a function of the relative coordinates $\vec{x}=\vec{x}_{2}-\vec{x}_{1}, t=t_{2}-t_{1}$. The modulus of $|\psi|$ normalized to its peak value has been plotted, rather than $|\psi|^{2}$, in order to enhance the visibility of the tails.

$t=t_{2}-t_{1}$ with respect to the idler photon, we note that for the considered BBO parameters:

(a) A signal photon will always arrive with a positive time delay $t>0$ with respect to its idler twin, since the group velocity of the signal (ordinary) wave $v_{1 g} \sim 1 / k_{1}^{\prime}$ is smaller than the group velocity of the idler (extraordinary) wave $v_{2 g} \sim$ $1 / k_{2}^{\prime}$.

(b) In the low-gain regime, coincidences are mainly produced by couples of photons generated from a single downconversion event (i.e., the contribution of photons generated by secondary stimulated processes to the measured coincidence rate $|\psi(\vec{x}, t)|^{2}$ is negligible $)$.

Because of this last condition, photon pairs are produced almost uniformly along the crystal through purely spontaneous PDC. Accordingly, the distribution of the delay times of the signal photons with respect to the idler photons at the crystal output face is nearly uniform within $t=0$ and the maximum delay time allowed by the length of the crystal $t_{\text {coh }}=\Omega_{0}^{-1}=$ $\left(l_{c} / v_{1 g}-l_{c} / v_{2 g}\right)$. It is important to stress that condition (b) is no more fulfilled in the high-gain regime, with $g \sim 1$ or larger, as stimulated PDC becomes the main source of photon pairs. We shall see indeed in the next section that by raising the parametric gain, the asymmetry of the biphoton amplitude along the temporal axis disappears.

Another distinctive feature of type-II PDC lies in that the diagonal tails that form the $\mathrm{V}$-shaped structure emerge only slightly from the overall structure [as it can be seen from Fig. 3(a)], since the coincidence rate $|\psi|^{2}$ does not vanish in the triangular region delimited by those tails [the region delimited by the dotted diagonals in Fig. 3(b). We shall see in Sec. VII that the contrast of those tails varies by changing the phasematching conditions and reaches its maximum for very large values of the collinear phase-mismatch parameter $\Delta_{0} l_{c}$. In the case of type-I PDC, on the contrary, the biphotonic structure appears as perfectly X-shaped, with the highest contrast of the tails obtained when $\Delta_{0} l_{c}$ is close to zero. Both in typeI and type-II PDC, the orientation of the tails in the $(x, t)$ plane is determined by the asymptotic behavior of the phasematching curve for large frequencies given by Eq. (27). To be more precise, the type-II biphotonic structure extends in the region with $t>0$ and $|x / t| \leqslant \Omega_{0}^{\prime \prime} / q_{0}$ delimited by the dotted diagonal lines indicated in Fig. 3(b) (a detailed explanation of this behavior will be given elsewhere [24]). 

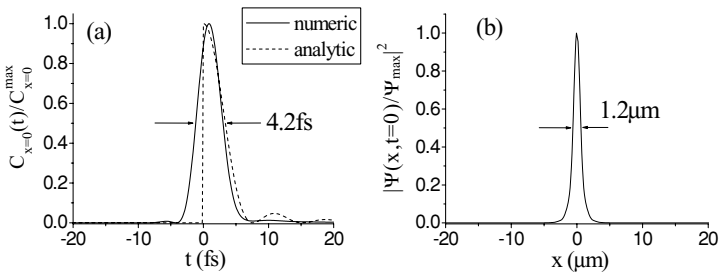

FIG. 4. Profile of the coincidence rate $|\psi(\vec{x}, t)|^{2}$ normalized to its peak value, along the temporal and the transverse $x$ axis, respectively [the cuts are indicated by the two white solid lines in Fig. 3(b)]. The FWHM of the correlation peaks (indicated in the figure) gives a measure of the relative localization of the biphotons in space and in time. The dashed line in (a) plots the analytical approximation given in Eq. (33b).

As for type-I PDC [12], an important feature of the biphotonic function is its strong temporal localization. This is best shown by the temporal profile of the coincidence rate $|\psi(\vec{x}, t)|^{2}$ for $\vec{x}=0$ illustrated in Fig. 4(a), which has a FWHM as narrow as $4.2 \mathrm{fs}$. Such an extreme two-photon localization is not linked to the characteristic coherence time $t_{\text {coh }}=\Omega_{0}^{-1}$, as it happens, for example, in the case of far-field measurements [25], but rather depends on the ultrabroad bandwidth of the whole PDC emission spectrum. This bandwidth can in principle extend over the whole frequency range up to the pump field frequency, though, in our case (and in any practical situation) it is limited by the frequency bandwidth $\Delta \Omega_{F}$ of the filter used in the numerical model. However, it is important to stress that such a localization is conditioned by our ability to resolve the photon spatial separation. Assuming that coincidences are measured by collecting all the photon in the transverse plane, without discriminating their relative position, the measured quantity corresponds to the spatially integrated joint-probability distribution,

$$
C(t)=\int d \vec{x}|\psi(\vec{x}, t)|^{2}=\int \frac{d \vec{q}}{(2 \pi)^{3}}\left|F_{12}(\vec{q}, t)\right|^{2},
$$

where the square modulus of the function,

$$
F_{12}(\vec{q}, t) \equiv \int \frac{d \Omega}{\sqrt{2 \pi}} e^{-i \Omega t} F_{12}(\vec{q}, \Omega),
$$

is proportional to the joint probability of finding a pair of photons with transverse momenta $\pm \vec{q}$ separated by the time interval $t$, obtained, for example, through a far-field measurement with two symmetrical pinholes in the positions corresponding to the opposite transverse wave vectors $\pm \vec{q}^{1}$. As already observed for type I [12], we see from this relation that $C(t)$ takes the form of an incoherent superposition of such probabilities and the temporal width of the latter represents

\footnotetext{
${ }^{1}$ Strictly speaking, the joint probability density of finding a signal and an idler photon separated by the time delay $t=t_{1}-$ $t_{2}$ and with the transverse wave vectors $\vec{q}$ and $\vec{q}^{\prime}$ is given by the square modulus of the function $\left\langle A_{1}\left(\vec{q}, t_{1}\right) A_{2}\left(\vec{q}^{\prime}, t_{2}\right)\right\rangle=\delta(\vec{q}+$ $\left.\vec{q}^{\prime}\right) \int \frac{d \Omega}{2 \pi} e^{-i \Omega\left(t_{1}-t_{2}\right)} F_{12}(\vec{q}, \Omega)$. The singularity arising from the presence of the delta function $\delta\left(\vec{q}+\vec{q}^{\prime}\right)$ is due to the PWP approximation and expresses the perfect transverse momentum correlation characterizing this limit.
}

therefore a lower bound for the temporal localization of the coincidence rate $C(t)$.

In contrast, if twin photons can be resolved spatially (e.g., by measuring coincidences in the same near-field positions), the measured quantity is given by the temporal profile of $|\psi|^{2}$ at $\vec{x}=0$,

$$
C_{x=0}(t) \equiv|\psi(\vec{x}=0, t)|^{2}=\left|\int \frac{d \vec{q}}{(2 \pi)^{5 / 2}} F_{12}(\vec{q}, t)\right|^{2},
$$

which behaves as a coherent superposition of the complex probability amplitudes $F_{12}(\vec{q}, t)$. Its temporal localization is by no means limited by the width $\sim t_{\mathrm{coh}}$ of the latter and the numerical result shown in Fig. 4(a) demonstrates that this is indeed the case.

In Appendix B, Eqs. (B6) and (B8), we performed complete analytical calculations based on the linear approximations (28) and (29) outlined in Sec. IV C. According to these results, the two coincidence rates are roughly given by the relations,

$$
\begin{gathered}
C(t) \propto \operatorname{Rect}\left[\frac{t-\frac{1}{2} t_{\mathrm{coh}}}{t_{\mathrm{coh}}}\right], \\
C_{x=0}(t) \propto \operatorname{sinc}^{2}\left[\frac{1}{2} \Delta \Omega_{\mathrm{F}}^{\prime} t\right] \operatorname{Rect}\left[\frac{t-\frac{1}{2} t_{\mathrm{coh}}}{t_{\mathrm{coh}}}\right],
\end{gathered}
$$

where $\operatorname{Rect}(x)$ denotes the rectangular function, equal to unity for $|x| \leqslant \frac{1}{2}$ and zero elsewhere, and $\Delta \Omega_{\mathrm{F}}^{\prime}$ indicates the bandwidth corresponding to the portion of the phase-matching curve selected by the frequency filter [see definition (B5) in Appendix B]. From these expressions we readily see that the temporal localizations characterizing the two coincidence rates may differ, at least for not too short crystals, by order of magnitudes. When the twin-photon positions are not discriminated, the coincidence rate temporal width is determined by the time delay that the photons accumulate in crossing the nonlinear medium because of GVM, that is $t_{\text {coh }}=l_{c} / v_{1 g}-l_{c} / v_{2 g}$, which for a few millimeters crystal is on the order of picoseconds. On the contrary, when the photon positions are discriminated (i.e., twin photons are collected at the same near-field positions) their typical correlation time is determined by the full PDC bandwidth intercepted in the measurement, $\Delta \Omega_{F}^{\prime}$. This one extends in principle over the optical frequency range and leads therefore to correlation times in the femtosecond range. These simple analytical results are confirmed by numerics. Figure 4(a) plots the analytical result (33b) (dashed line) together with the more accurate numerical result (solid line). The predicted FWHM value for $C_{x=0}(t)$ derived from Eq. (33b) (i.e., $t_{1 / 2}=2.78 / \Delta \Omega_{F}^{\prime}=$ $3.7 \mathrm{fs}$ ), underestimates the numerical evaluation by about $10 \%$, as it can be inferred from Fig. 4(a) where the numerical and the analytical estimates of $C_{x=0}(t)$ are plotted. The smoother and broader shape of the numerical result (solid line) is essentially due to GVD effects which are neglected in the analytical calculations. The scaling with $1 / \Delta \Omega_{F}^{\prime}$ has been fully verified through the numerical simulations.

Figure 5(b) plots the integrated coincidence rate $C(t)$ and compares the analytical result (dashed line) with the numerics (solid line). Again, the slightly smoother and broader profile of the numerical result is due to GVD effects neglected in the analytics. A more accurate analytical estimation can be done 

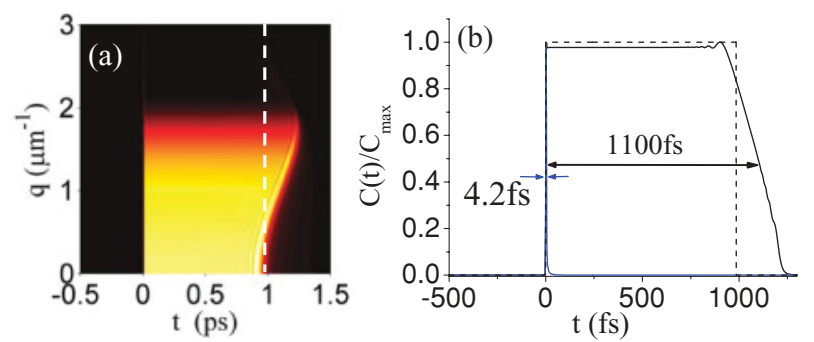

FIG. 5. (Color online) (a) Plot of $\left|F_{12}(\bar{q}, t)\right|$; the vertical dashed line corresponds to the coherence time $t_{\mathrm{coh}}=985 \mathrm{fs}$ associated to temporal walk-off. (b) Spatially integrated coincidence rate $C(t)$ normalized to its peak value (solid line). The dashed line corresponds to the analytical approximation (33a). The 4.2-fs narrow peak $C_{x=0}(t)$ is also shown in the figure for comparison.

on the basis of the quadratic approximation of Sec. IV B and will be presented elsewhere [26].

Figure 5(a) displays the numerical evaluation of $\left|F_{12}(\bar{q}, t)\right|$ in the plane $(t, \bar{q})$. As predicted by Eq. (B1) of Appendix B, its cross section for a given value of $\bar{q}$ has a box-like shape extending from $t=0$ up to an upper bound on the order of $t_{\text {coh }}=\Omega_{0}^{-1}$. The slow dependence on $\bar{q}$ of this upper bound is related to the dispersion of the GVM, which is not taken into account in the simplified model that led to the approximations (B1) and (33a) for $F_{12}(\bar{q}, t)$ and $C(t)$ (see Appendix B for more details).

\section{TRANSITION TO THE HIGH-GAIN REGIME}

In this section we investigate numerically the behavior of the biphoton amplitude for increasing values of the parametric gain. As can be seen from Fig. 6, when $g$ is raised, a V-shaped structure that mirrors that in the $t>0$ half-plane develops in the $t<0$ half-plane. Thus, the strong asymmetry along the temporal axis that characterizes the low-gain regime [see Fig. 6(a)] progressively disappears and a transition from a $\mathrm{V}$-shaped to an X-shaped structure is observed. This can also be inferred from the behavior of the integrated correlation function $C(t)$ plotted for $g=1.2$ and $g=4$ in Figs. 7(a) and 7(b), respectively (solid line). Its shape transforms progressively from the box-like coincidence rate obtained in the low-gain regime [see Fig. 5(b)] to a bell-shaped curve whose position of the maximum $t_{\text {shift }}$ shifts toward zero for increasing gains [Figs. 7(a) and 7(b)]. Figure 8 plots the cross section of the biphoton correlation, $C_{x=0}(t)$, for the same gain values. Also in this case the shift toward positive time delays decreases at high parametric gains, and the correlation peak becomes almost symmetric.

We can explain qualitatively this behavior noticing that the mean number of down-converted photons grows exponentially with the crystal length in a regime of high parametric gain, as the produced photon pairs stimulate the generation of many other photon pairs in a cascading process. The "slow" signal photons can thus be generated in processes stimulated by the "faster" idler photons and vice versa, so that the arrival time distributions of the signal and the idler photons tend to overlap when this cascading effect becomes the dominant source of photon pairs. As a result, the strong asymmetry of the correlation function along the temporal axis, that
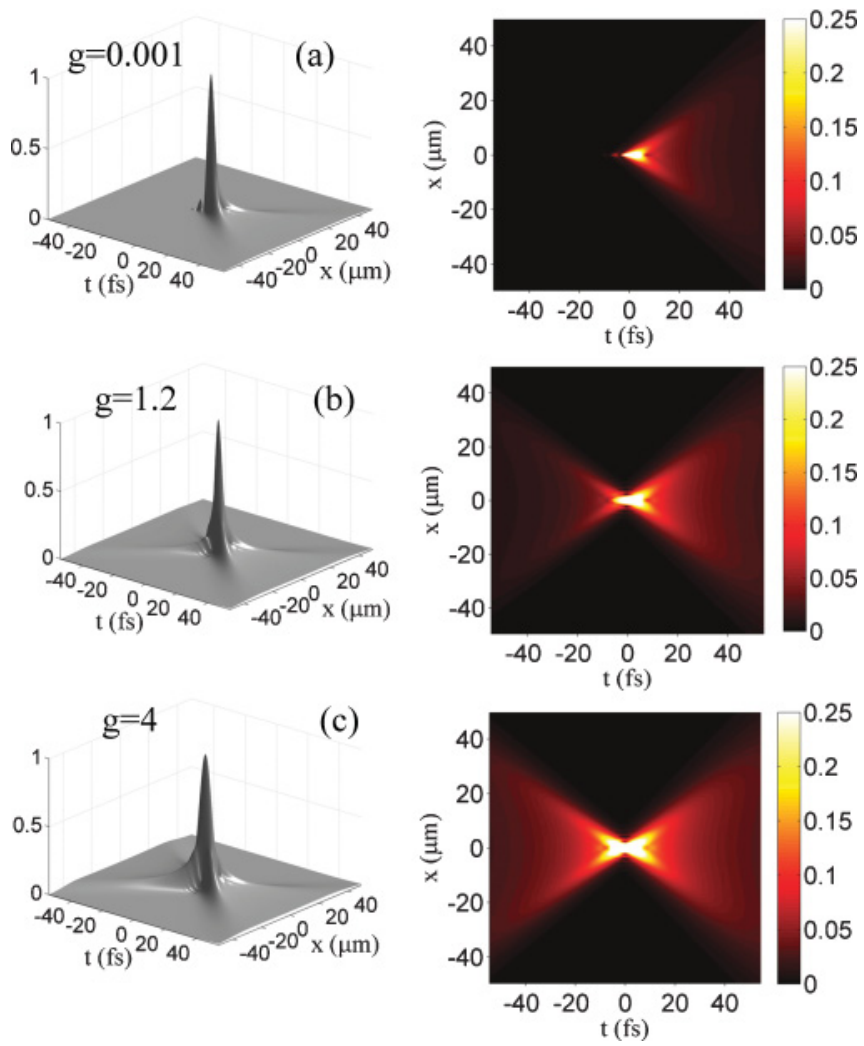

FIG. 6. (Color online) Behavior of the biphoton correlation for increasing value of the parametric gain (from top to bottom). The modulus of $\psi$ normalized to its peak value is plotted in the $(x, t)$ plane. The color map of the $2 \mathrm{D}$ plots (right) is truncated to $1 / 4$ of the peak value of $|\psi|$ to enhance the contrast of the tails.
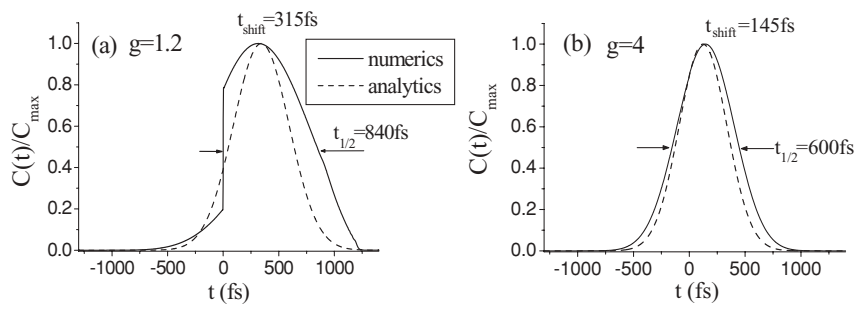

FIG. 7. Plot of the spatially unresolved coincidence rate $C(t)$ normalized to its peak value for (a) $g=1.2$ and (b) $g=4$. Its FWHM width $t_{1 / 2}$ and the temporal delay at the peak value $t_{\text {shift }}$ are indicated in the figure. The dashed line reproduces the Gaussian approximation given in Eq. (40a).

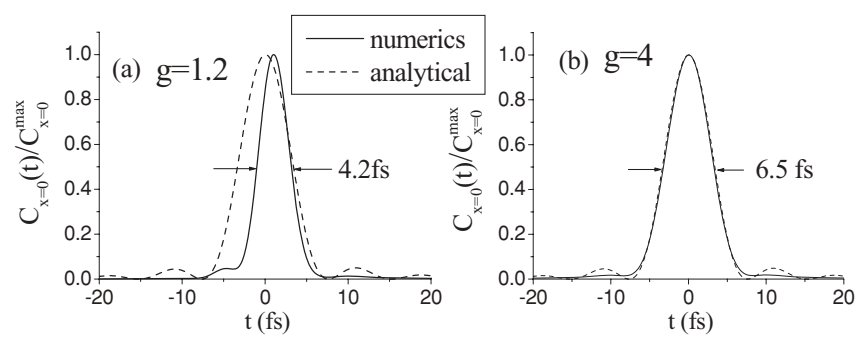

FIG. 8. Plot of coincidence rate at the same near-field position, $C_{x=0}(t)$ normalized to its peak value for (a) $g=1.2$ and (b) $g=4$. Its FWHM is indicated in the figure. The dashed line reproduces the analytical approximation given in Eq. (40b). 
reveals the temporal delay of the "slow" signal photons with respect to the idler photons in the low-gain regime, disappears progressively when the parametric gain is raised.

From a more quantitative point of view, we can gain some insight by investigating the behavior of the probability amplitude $F_{12}(\vec{q}, t)$ defined in Eq. (31) for increasing gain values. We first notice that the phase of $F_{12}(\vec{q}, \Omega)$ close to perfect phase matching, where $\left|F_{12}(\vec{q}, \Omega)\right|$ is not negligible, is well approximated by the expression,

$$
\arg \left[F_{12}(\vec{q}, \Omega)\right] \approx \frac{\tanh g}{2 g} \Delta(\vec{q}, \Omega) l_{c} \approx \frac{\tanh g}{2 g} \frac{\Omega-\Omega^{(L)}(\bar{q})}{\Omega_{0}},
$$

where in the last equality we used the linearized approximations (28) and (29) for the phase-mismatch function. By extending this approximation to the whole $\Omega$ axis and performing the substitution $\Omega \rightarrow \Omega+\Omega^{(L)}(\bar{q})$ for the integration variable, the Fourier transform (31) can then be written in the form,

$$
F_{12}(\bar{q}, t) \approx e^{-i \Omega^{(L)}(\bar{q}) t} \int \frac{d \Omega}{\sqrt{2 \pi}} e^{-i \Omega\left[t-\frac{\tanh g}{2 g} t_{\mathrm{coh}}\right]} \tilde{h}(\Omega),
$$

where

$$
\tilde{h}(\Omega)=\left|F_{12}\left(\bar{q}, \Omega^{(L)}(\bar{q})+\Omega\right)\right|
$$

does not depend on $\vec{q}$, since, thanks to the change of variable, it is a function of $\Delta\left(\bar{q}, \Omega^{(L)}(\bar{q})+\Omega\right) l_{c}=\Omega / \Omega_{0}$ alone, as can be inferred from Eqs. (11), (28) and (29). Therefore, the square modulus of $F_{12}(\bar{q}, t)$ depends only on the temporal argument $t-\frac{\tanh g}{2 g} t_{\text {coh }}$ and can be written as

$$
\left|F_{12}(\vec{q}, t)\right|^{2}=\left|h\left(t-\frac{\tanh g}{2 g} t_{\mathrm{coh}}\right)\right|^{2},
$$

where $h(t)$ denotes the inverse Fourier transform of $\tilde{h}(\Omega)$. Since $\tilde{h}(\Omega)$ is a real function, $|h(t)|$ is accordingly an even function of its argument and the same holds true for the integrated coincidence rate defined in Eq. (30):

$$
C(t)=\int_{R_{\vec{q}}} d \vec{q}\left|F_{12}(\vec{q}, t)\right|^{2} \propto\left|h\left(t-\frac{\tanh g}{2 g} t_{\mathrm{coh}}\right)\right|^{2} .
$$

We obtain a finite quantity, as we limited the integration in $\vec{q}$ space to the finite region $R_{\vec{q}}$ defined in Appendix B, Eqs. (B2) and (B3), corresponding to the portion of the phase-matching curve selected by the temporal frequency filter. Equation (38) tells us that $C(t)$ is evenly distributed in time around $t_{\text {shift }}=$ $\frac{\tanh g}{2 g} t_{\mathrm{coh}}$, with a temporal width on the order of $t_{\mathrm{coh}}=\Omega_{0}^{-1}$, the inverse of the typical variation scale of $\tilde{h}(\Omega)$ on the $\Omega$ axis. For low parametric gains $t_{\text {shift }}$ takes its maximum value [i.e., $\left.t_{\text {shift }} \rightarrow \frac{t_{\text {coh }}}{2}=\frac{1}{2}\left(l_{c} / v_{1 g}-l_{c} / v_{2 g}\right)\right]$. Notice that this temporal value corresponds to the average time delay of the twin photons at the crystal output face assuming that they are generated uniformly along the crystal length, as is the case in the purely spontaneous regime of PDC. On the other hand, this shift toward positive time delays $t_{\text {shift }}$ decreases to zero as the parametric gain is raised and the stimulated regime of PDC is reached (i.e., for $g$ well above unity). The distribution of $C(t)$ becomes, therefore, more and more symmetric around $t=0$ and the same holds true for the temporal behavior of the functions $F_{12}(\vec{q}, t)$ and $\psi(\vec{x}, t)$, as can be seen from the numerical examples illustrated in Fig. 6.

In contrast, the temporal width of the correlation function with resolved positions, $C_{x=0}(t)$, is not determined by the variation scale $\Omega_{0}$ of $\tilde{h}(\Omega)$. As in the low-gain regime, the phase factor that depends on $\bar{q}$ in the expression (35) play a fundamental role in the evaluation of the coherent superposition of the amplitudes $F_{12}(\vec{q}, t)$ that defines $C_{x=0}(t)$ in Eq. (32). Substituting Eq. (35) into (32) and using the identity (B7) derived in Appendix B, we find

$$
\begin{aligned}
C_{x=0}(t) & =\left|\int_{R_{\vec{q}}} \frac{d \vec{q}}{(2 \pi)^{5 / 2}} e^{-i \Omega^{(L)}(\bar{q}) t}\right|^{2} \times\left|h\left(t-\frac{\tanh g}{2 g} t_{\mathrm{coh}}\right)\right|^{2} \\
& \propto \operatorname{sinc}^{2}\left(\frac{\Delta \Omega_{F}^{\prime} t}{2}\right) C(t) .
\end{aligned}
$$

The width of $C_{x=0}(t)$ is thus determined by the intercepted PDC bandwidth $\Delta \Omega_{\mathrm{F}}^{\prime}$ as in the low-gain regime [see Eq. (33b)], while the broader function $C(t)$ acts here only as a slow modulation, since we are always assuming that $\Delta \Omega_{\mathrm{F}}^{\prime} \gg \Omega_{0}$. The strong temporal localization of the two-photon correlation in the same near-field position is, therefore, robust against a rise of the parametric gain. The more accurate numerical evaluation shown in Figs. 8(a) and 8(b) (solid line) confirms this result: we observe only a slight increase of the FWHM correlation time, by about $50 \%$ in the worst case. The two orders of magnitude improvement with respect to the localization of $C(t)$ persists, therefore, at high parametric gains, as can be inferred by comparing Figs. 7 and 8 (the corresponding FWHM are indicated in the figure).

In order to obtain an explicit expression for $h(t)$, we verified that $\left|F_{12}(\vec{q}, \Omega)\right|$, seen as a function of $\Delta(\vec{q}, \Omega) l_{c}$, can be replaced with the Gaussian fit $\left|F_{12}(\Delta=0)\right| \exp \left[-\left(\Delta l_{c}\right)^{2} / \delta_{g}^{2}\right]$ for $g \gtrsim$ 1.5 , the fitting parameter $1 / \delta_{g}^{2}=(1 / \tanh g-1 / g) / 4 g$ being obtained by matching the second-order derivative for $\Delta=$ 0 . We obtain thereby the following expressions for $C(t)$ and $C_{x=0}(t)$ :

$$
\begin{gathered}
C(t) \propto e^{-\frac{\delta_{g}^{2}}{2 t_{\mathrm{coh}}^{2}}\left[t-\frac{\mathrm{tanh} g}{2 g} t_{\mathrm{coh}}\right]^{2}}, \\
C_{x=0}(t) \propto e^{-\frac{\delta_{g}^{2}}{2 t_{\mathrm{coh}}^{2}}\left[t-\frac{\tanh g}{2 g} t_{\mathrm{coh}}\right]^{2}} \operatorname{sinc}^{2} \frac{\Delta \Omega_{\mathrm{F}}^{\prime} t}{2} .
\end{gathered}
$$

Although these analytical approximations fail to reproduce the exact shape of the correlation functions at intermediate gains, they provide a good description of their overall behavior for increasing values of $g$. Figures 7 and 8 plot these analytical curves (dashed lines) for comparison with the more accurate numerical evaluations (solid lines).

According to Eq. (40a), the integrated coincidence rate $C(t)$ becomes at high gains almost Gaussian, centered around $t_{\text {shift }}$ and with a FWHM $t_{1 / 2}$ given by

$$
\begin{gathered}
t_{\text {shift }}=\frac{\tanh g}{2 g} t_{\mathrm{coh}} \approx t_{\mathrm{coh}} / 2 g, \\
t_{1 / 2}=2.35 t_{\mathrm{coh}} / \delta_{g} \approx 1.177 t_{\mathrm{coh}} / \sqrt{g},
\end{gathered}
$$

the last expressions on the right-hand side giving the asymptotic limit at high parametric gains. The numerical estimation 


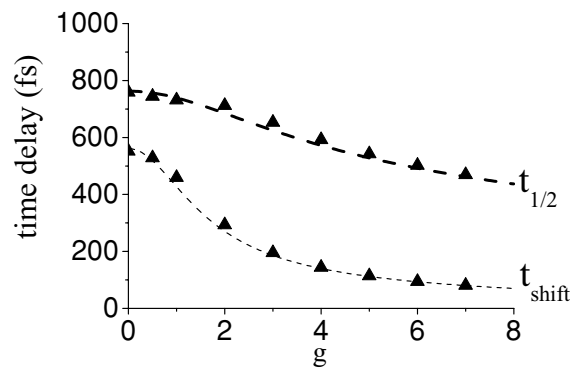

FIG. 9. The numerical evaluation of the mean temporal shift toward positive time delays, $t_{\text {shift }}$, and the width $t_{1 / 2}$ of $C(t)$, are plotted as a function of the parametric gain (triangles). The dashed lines are obtained by fitting the predicted scaling behavior given by Eqs. (41a) and (41b).

of these parameters at different gain values (triangles) is fitted with the predicted behavior (dashed lines) in Fig. 9. In this plot the discrete data have been obtained from the numerical estimation of $C(t)$ by evaluating the mean and the standard deviation of the normalized coincidence rate $C(t) / \int C(t) d t$, respectively. Notice that the analytical curves (41) have been fitted by treating the walk-off time $t_{\mathrm{coh}}$ as a fitting parameter, obtaining a value about 1.25 times larger than its value for $\Omega=0$, namely $\Omega_{0}^{-1}=985$ fs. It can indeed be verified that the walk-off time of the best phase-matched modes slowly increases with the transverse wave vector $\bar{q}$. By ignoring this variation in the analytical treatment (see Appendix A), the values of $t_{\text {shift }}$ and $t_{1 / 2}$ are therefore slightly underestimated [this can also be inferred from the dashed curve in Fig. 7 which reproduces the Gaussian approximation (40a) with $\left.t_{\mathrm{coh}}=985 \mathrm{fs}\right]$.

The stronger localization of $C(t)$ at high gains can be explained noticing that in such a regime most photon pairs are produced through stimulated PDC close to the crystal end face. The mean temporal walk-off undergone by the twin photons during propagation in the birefringent media, the main source of broadening for $C(t)$, is thus reduced with respect to the purely spontaneous (low-gain) regime. However, because of the slow decrease of $t_{1 / 2} \propto 1 / \sqrt{g}$ [see Eq. (41b)], it still remains on the order of $t_{\text {coh }} \sim 1$ ps for realistic gain values.

On the other hand, the faster decrease of $t_{\text {shift }} \approx t_{\text {coh }} / 2 g$ [see Eq. (41a)] is sufficient to make the asymmetry of both $C(t)$ and $|\psi(\vec{x}, t)|^{2}$ diminish substantially, as it can be inferred from the numerical result shown in Fig. 6 and from the temporal profile shown in Figs. 7 and 8.
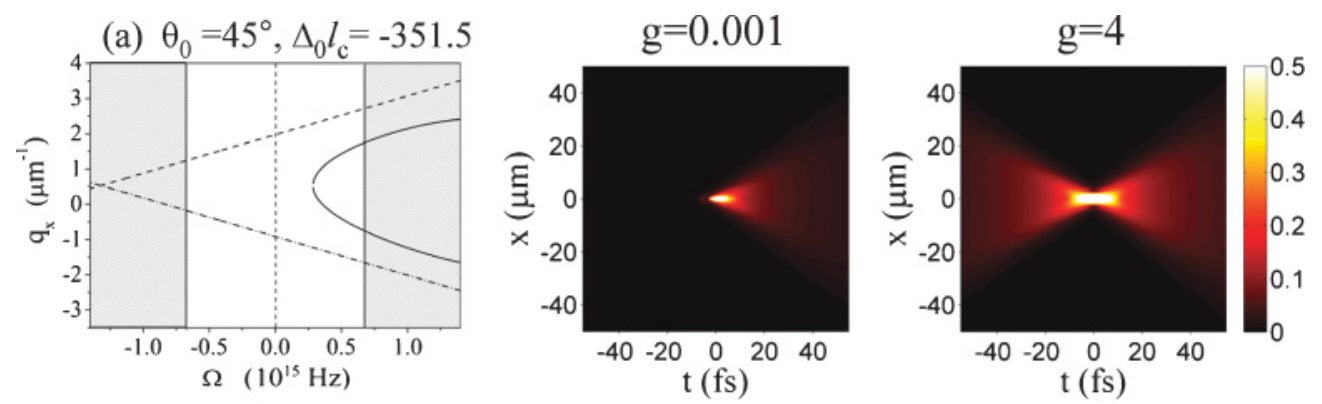

(b) $\theta_{0}=50^{\circ}, \Delta_{0} l_{\mathrm{c}}=20.6$
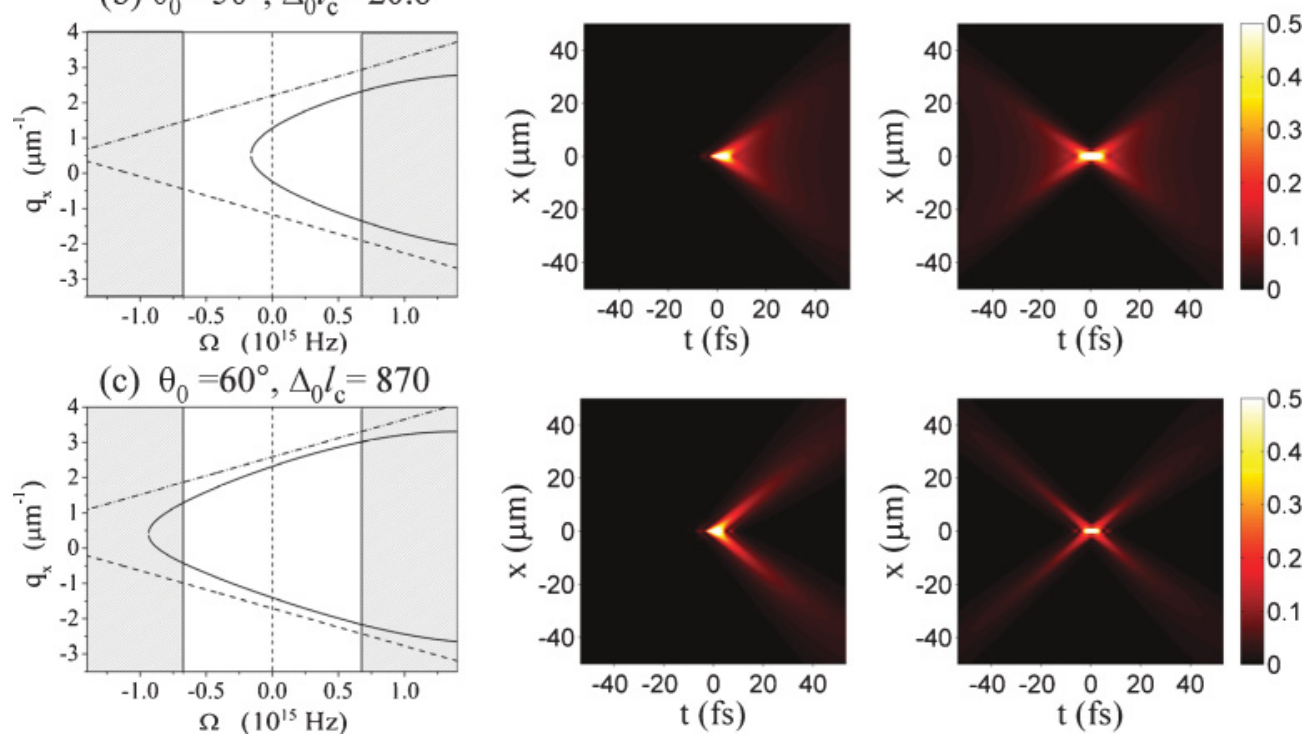

FIG. 10. (Color online) (Top to bottom) The collinear phase-mismatch parameter $\Delta_{0} l_{c}$ is raised by increasing $\theta_{0}$ (the values are indicated in the figure). The unshaded region indicates the frequency bandwidth selected by the super-Gaussian filter used in the simulation. (Left) The phase-matching curve shifts toward negatives $\Omega$; the dashed lines reproduce the asyntoptes given in Eq. (27). (Middle and right) $|\psi|$ is plotted in the $(x, t)$ plane for $g=0.001$ and $g=4$, respectively. As $\Delta_{0} l_{c}$ is raised, the contrast of the tails of the V-shaped (or X-shaped) structure improves since the contribution of the phase-matched modes close to the asymptotes increases. Note that the $|\psi|$ has been truncated to $1 / 2$ of its peak value to enhance the visibility of the tails. 


\section{BEHAVIOR OF THE BIPHOTON AMPLITUDE FOR DIFFERENT CRYSTAL TUNING ANGLES}

In this section we investigate the behavior of the biphoton correlation in the space-time domain with respect to changes in the pump axis orientation with respect to the crystal axis. In the type-II BBO case we are considering, by increasing the pump axis incidence angle $\theta_{0}$, the collinear phase-mismatch value $\Delta_{0} l_{c}$ becomes more and more positive. The central and right panels in Fig. 10 plot the biphotonic structure obtained for increasing values of $\theta_{0}$ and $\Delta_{0} l_{c}$, for $g=0.001$ and $g=4$, respectively. We see that the contrast of the diagonal tails along the lines $t= \pm \frac{q_{0}}{\Omega_{0}^{\prime \prime}} x$ is strongly enhanced and its $\mathrm{V}$ like (or X-like) shape becomes more distinguishable as $\Delta_{0} l_{c}$ increases (from top to bottom). The strongest $x$ - $t$ relationship is thus obtained for large noncollinear phase-mismatch values, in contrast to the case of type-I PDC, where it occurs for $\Delta_{0} l_{c}=0[12,14]$.

In the Fourier domain, this feature is reflected in the behavior of the phase-matching curve (Fig. 10, left, solid line), which for increasing values of $\Delta_{0} l_{c}$ approaches more and more the asymptotic behavior given by Eq. (27) (Fig. 10, dashed lines) within the region selected by the frequency filter (delimited by the gray regions). The temporal frequency $\Omega_{\text {tp }} \approx \Omega_{+}(\bar{q}=0)$ corresponding to the turning point of the hyperbolic branch given by Eq. (26) moves indeed toward large negative values as $\Delta_{0} l_{c}$ becomes more and more positive. It may be shown [24] that under those conditions, the ratio between the transverse spatial separation $\Delta x$ and the temporal delay $\Delta t$ of emitted twin photons approaches the constant value $q_{0} / \Omega_{0}^{\prime \prime}$ for all the collected frequencies, a feature which explains the highest contrast of the tails displayed by the biphotonic structure in the $(x, t)$ plane obtained for large values of $\Delta_{0} l_{c}$ [see Fig. 10(c), middle and left].

The situation is different in type-I PDC, where the absence of GVM leads to the symmetric phase-matching curve $\Omega_{ \pm}(q)= \pm \Omega_{0} \sqrt{\frac{q^{2}}{q_{0}^{2}}-\Delta_{0} l_{c}}$, as can be inferred from the quadratic approximation (23) by taking the limit $\Omega_{0} \rightarrow \infty$, $\rho_{2}=0$. In this case, the ratio $\Delta x / \Delta t$ is close to $\Omega_{0}^{\prime \prime} / q_{0}$ for all frequencies when the collinear phase-mismatch parameter is equal to zero [14,24]. In type-I PDC the perfectly X-shaped geometry of the biphotonic correlation occurs, therefore, for $\Delta_{0} l_{c}=0$.

\section{CONCLUSIONS}

We have shown that the spatiotemporal structure of the biphoton amplitude in type-II phase matching exhibits interesting properties that qualitatively differ from those of type-I PDC. We verified that in the coincidence regime the signal-idler GVM generates an asymmetry along the temporal dimension which progressively disappears as the parametric gain is increased. Indeed, the purely spontaneous PDC regime is characterized by a V-shaped structure that extends only in the half-plane corresponding to the "slow" signal photons arriving with positive time delays with respect to their "fast" idler twins. As the parametric gain is raised and stimulated PDC becomes the dominant source of photon pairs, we observe a transition from this V-shaped geometry toward a symmetric X-shaped structure that includes both positive and negative time delay.
By using an approximated model that neglects GVD, we have been able to quantify analytically how the asymmetry along the temporal axis progressively disappears as the parametric gain increases.

On the other side, according to our analysis the localization in space and time of this structure is comparable to that found in a type-I PDC and is robust with respect to increases of the parametric gain. In particular, we evaluated the correlation times of the PDC field, by considering both measurements that collect the photons on the whole transverse plane without spatial resolution and measurements able to discriminate the relative near-field positions of the twin photons. In the first case the relative temporal localization is determined by the group velocity mismatch that characterizes type-II PDC and cannot go below the picosecond range for usual crystal lengths of a few millimeters. In the second case, on the contrary, it is determined by the full emission bandwidth allowed by the phase-matching mechanism and is therefore unrelated to the length of the crystal. An improvement of more than two orders of magnitude can, therefore, be obtained for the temporal localization by resolving the twin photon spatially. Approximated analytical expressions for both the spatially resolved and the spatially unresolved temporal correlation have been obtained within a simplified model.

\section{ACKNOWLEDGMENT}

We acknowledge financial support from the Future and Emerging Technologies (FET) program within the Seventh Framework Program for Research of the European Commission, under the FET-Open grant agreement HIDEAS (Grant No. FP7-ICT-221906).

\section{APPENDIX A: APPROXIMATION OF THE PHASE-MISMATCH FUNCTION $\Delta(\vec{q}, \Omega) l_{c}$}

In a type-II phase-matching configuration, the phasemismatch function $\Delta(\vec{q}, \Omega) l_{c}$ expressed by Eq. (12) lacks the radial symmetry with respect to the transverse wave vector $\vec{q}$ that characterizes type-I PDC. In this appendix we shall briefly illustrate the origin of the approximate expression (16) that allows us to express the biphoton amplitude (10) as a Hankel transformation with respect to the spatial frequency coordinates [see Sec. IV A, Eq. (21)]. We also show that for the chosen crystal orientation it provides a good fit of the "exact" phase-mismatch function over a very broad range of temporal frequencies [i.e., that which can be obtained by evaluating numerically the longitudinal $k$-vector $k_{j z}(\vec{q}, \Omega)$ with an iterative algorithm]. The starting point is the paraxial approximation which assumes that $q \ll k_{j}(\vec{q}, \Omega)$ so that we can write

$$
k_{j z}(\vec{q}, \Omega) \approx k_{j}(\vec{q}, \Omega)-\frac{q^{2}}{2 k_{j}(\vec{q}, \Omega)} .
$$

Consistently, we shall now perform the Taylor expansion of the wave numbers $k_{j}(\vec{q}, \Omega)$ up to second order in the transverse wave-vector components $q_{x}$ and $q_{y}$ around $\vec{q}=0$, neglecting all the higher-order terms. We now focus on the uniaxial BBO crystal cut for type-II (e-oe) phase matching considered in the numerics. While the ordinary wave $(j=1$ in 


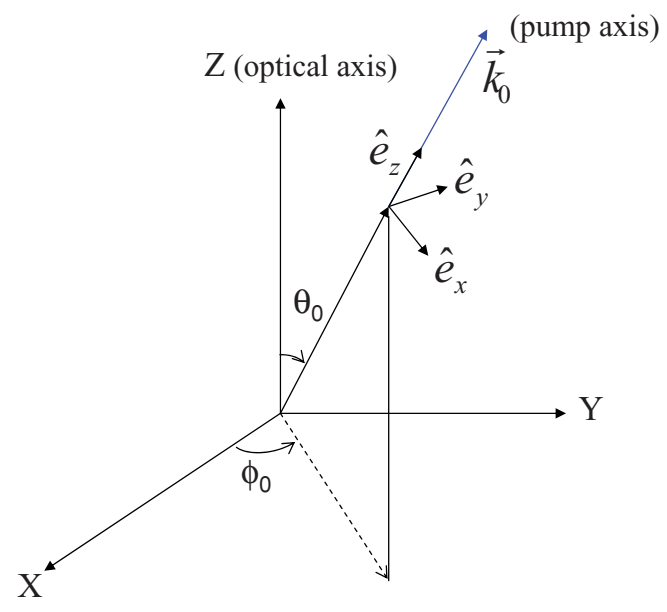

FIG. 11. Orientation of the pump field reference frame $(x, y, z)$ (indicated by the three unit vectors $\hat{e}_{x}, \hat{e}_{y}, \hat{e}_{z}$ ) with respect to the crystal axis reference frame $(X, Y, Z)$. The pump axis direction angles $\theta_{0}, \phi_{0}$ are also indicated in the figure.

our case) propagates isotropically so that $k_{1}(\Omega) \equiv \frac{\omega_{s}+\Omega}{c} n_{o}(\Omega)$ does not depend on $\vec{q}$, the same is not true for the pump $(j=0)$ and the idler $(j=2)$ field wave numbers which are both extraordinarily polarized. Let us denote with $\left(\theta_{0}, \phi_{0}\right)$ the direction angles of the pump $k$ vector (i.e., the $z$ axis) with respect to the crystal principal axis reference frame $(X, Y, Z)$ (see Fig. 11). For a given transverse wave vector $\vec{q} \equiv\left(q_{x}, q_{y}\right)$ and a given wavelength $\lambda=2 \pi c /\left(\omega_{s}+\Omega\right)$ of the idler wave, the corresponding direction angles $(\theta, \phi)$ in the $(X, Y, Z)$ reference frame are related to $\vec{q}$ and $\Omega$ through the implicit relations,

$$
\begin{gathered}
q_{x}=k_{2}(\theta, \Omega)\left[\sin \theta \cos \theta_{0} \cos \left(\phi-\phi_{0}\right)-\cos \theta \sin \theta_{0}\right], \\
q_{y}=k_{2}(\theta, \Omega) \sin \theta \sin \left(\phi-\phi_{0}\right),
\end{gathered}
$$

where we assumed the $x$ axis lies in the $z-Z$ optics plane determined by the pump axis and optical axis directions as shown in Fig. 11 (following the conventions used in [18]).

Dealing with uniaxial crystals, the idler wave number $k_{2}(\theta, \lambda)=\frac{\omega_{2}+\Omega}{c} n_{2}(\theta, \lambda)$ does not depend on the azimuthal angle $\phi$ and the corresponding refractive index varies with $\theta$ according to the relation [27],

$$
n_{2}^{2}(\theta, \lambda)=\frac{n_{e}^{2}(\lambda)}{1-\gamma(\lambda) \cos ^{2} \theta},
$$

where $n_{o}(\lambda)$ and $n_{e}(\lambda)$ are the ordinary and extraordinary principal axis refraction indexes and $\gamma(\lambda) \equiv 1-n_{e}^{2}(\lambda) / n_{o}^{2}(\lambda)$ is a positive parameter as we are considering a negative crystal (for which $n_{e}<n_{o}$ ). In the numerics we used the Sellmeier dispersion formula found in Ref. [18], which provides good approximations for $n_{o}(\lambda)$ and $n_{e}(\lambda)$ over a broad range of frequencies. Using Eqs. (A2)-(A4) we now evaluate the derivatives of $k_{2}$ with respect to $q_{x}$ and $q_{y}$ up to second order along the $z$-axis direction (i.e., for $\vec{q}=0$ ). The first order derivative with respect to $q_{y}$ is equal to zero while

$$
\left.\frac{\partial k_{2}}{\partial q_{x}}\right|_{\vec{q}=0, \Omega}=-\frac{\gamma(\Omega) \sin \theta_{0} \cos \theta_{0}}{1-\gamma(\Omega) \cos ^{2} \theta_{0}} \equiv-\rho_{2}(\Omega),
$$

gives the walk-off angle $\rho_{2}$ of the idler wave along the pump axis direction. The nonvanishing second-order derivatives can be written as

$$
\begin{gathered}
\left.\frac{\partial^{2} k_{2}}{\partial q_{x}^{2}}\right|_{\vec{q}=0, \Omega}=\frac{1}{k_{2}(\vec{q}=0, \Omega)}\left(\rho_{2}^{2}(\Omega)-\frac{2 \rho_{2}(\Omega)}{\tan 2 \theta_{0}}\right), \\
\left.\frac{\partial^{2} k_{2}}{\partial q_{y}^{2}}\right|_{\vec{q}=0, \Omega}=\frac{-1}{k_{2}(\vec{q}=0, \Omega)} \frac{\rho_{2}(\Omega)}{\tan \theta_{0}} .
\end{gathered}
$$

These expressions can be used to perform the Taylor expansion of $k_{2}(\vec{q}, \Omega)$ in the paraxial approximation (A1), keeping terms up to second order in $q_{x}$ and $q_{y}$. The corresponding quadratic expansion of the phase-mismatch function (12) can then be written in the form,

$$
\begin{aligned}
\Delta(\vec{q}, \Omega) \equiv & k_{1 z}(\Omega)+k_{2 z}(-\vec{q},-\Omega)-k_{0} \approx k_{1}(\Omega) \\
& +k_{2}(\vec{q}=0,-\Omega)-k_{0}-\left.\frac{\partial k_{2}}{\partial q_{x}}\right|_{\vec{q}=0,-\Omega} q_{x} \\
& +\left.\frac{1}{2} \frac{\partial^{2} k_{2}}{\partial q_{x}^{2}}\right|_{\vec{q}=0,-\Omega} q_{x}^{2}+\left.\frac{1}{2} \frac{\partial^{2} k_{2}}{\partial q_{y}^{2}}\right|_{\vec{q}=0,-\Omega} q_{y}^{2} \\
& -\frac{1}{2 k_{1}(\Omega)} q^{2}-\frac{1}{2 k_{2}(\vec{q}=0,-\Omega)} q^{2},
\end{aligned}
$$

where we made explicit the independence of $k_{1 z}$ on $\vec{q}$ and kept only the nonvanishing derivatives. We obtain the phase-mismatch function in the form given in Eqs. (16)-(19) by substituting the explicit expressions of the second-order derivatives (A6) into Eq. (A7) and by rescaling the Fourier coordinates according to Eq. (17). By neglecting the slow dependence on $\Omega$ of the dimensionless parameters $\beta_{x}, \beta_{y}$ given in Eq. (19) (i.e., by taking their values at $\Omega=0$ ), the time consumption of the numerical algorithm is drastically reduced. We verified numerically that this latter approximation does not affect substantially the precision of the calculation.

\section{APPENDIX B: EVALUATION OF $C(t)$ AND $C_{x=0}(t)$ AT LOW GAINS}

In this appendix we use the parabolic approximation for the phase-matching function presented in Sec. IV C in order to obtain analytical expressions for the coincidence rates $C(t)$ and $C_{x=0}(t)$ in the low-gain regime. By considering a frequency region such that $\left|\Omega / \Omega_{0}\right| \ll 1 / \epsilon$, GVD can be neglected and we can use the approximated phase mismatch function given by Eq. (28). Substituting this relation in the low-gain limit expression of $F_{12}(\bar{q}, \Omega)$ given by Eq. (15a) we can evaluate its inverse Fourier transform with respect to the temporal frequency coordinate defined in Eq. (31), obtaining, thereby,

$$
F_{12}(\bar{q}, t)=\sqrt{2 \pi} g \Omega_{0} e^{-i \Omega^{(L)}(\bar{q}) t} \operatorname{Rect}\left[\frac{t-\frac{1}{2} t_{\mathrm{coh}}}{t_{\mathrm{coh}}}\right],
$$

where $\operatorname{Rect}(x)$ denotes the rectangular function, equal to unity for $|x| \leqslant \frac{1}{2}$ and zero elsewhere, $t_{\mathrm{coh}}=\Omega_{0}^{-1}$ is the characteristic walk-off time, and $\Omega_{+}^{L}(\bar{q})$ is the parabolic approximation for the phase-matching curve given in Eq. (29). In order to provide a finite integration domain in $\vec{q}$ space for the integral that defines $C(t)$ and $C_{x}(t)$ in Eqs. (30) and (32), 
we now consider a box-shaped temporal frequency filter that selects the frequency interval $-\frac{1}{2} \Delta \Omega_{F}<\Omega<\frac{1}{2} \Delta \Omega_{F}$ around degeneracy. Accordingly, we restrict the domain of integration to the region $R_{\vec{q}}$ obtained by projecting the portion of the phase-matching surface $\Delta_{L}(\bar{q}, \Omega)=0$ selected by the frequency filter into the plane $\left(q_{x}, q_{y}\right)$. We obtain thereby the annular region centered on $\left(q_{C}, 0\right)$,

$$
R_{\vec{q}}=\left\{\left(q_{x}, q_{y}\right) \in \mathbb{R}^{2} ; \bar{q}_{\min } \leqslant \bar{q} \leqslant \bar{q}_{\max }\right\},
$$

with $\bar{q} \equiv \sqrt{\left(q_{x}-q_{C}\right)^{2}+q_{y}^{2}}$ and

$$
\begin{gathered}
\bar{q}_{\min }=q_{0} \sqrt{\max \left\{D_{0}-\frac{\Delta \Omega_{F}}{2 \Omega_{0}}, 0\right\}}, \\
\bar{q}_{\max }=q_{0} \sqrt{D_{0}+\frac{\Delta \Omega_{F}}{2 \Omega_{0}}} .
\end{gathered}
$$

If $D_{0}=\Delta_{0} l_{c}+\frac{1}{4} \bar{k} l_{c} \rho_{2}^{2}>\Delta \Omega_{F} / 2 \Omega_{0}$, the boundary values $\bar{q}_{\text {min }}$ and $\bar{q}_{\text {max }}$ are both positive and correspond to the real solutions of the equation $\Delta_{L}\left(\bar{q}, \pm \frac{\Delta \Omega_{F}}{2}\right) l_{c}=0$. This corresponds in fact to the case illustrated in Fig. 10(c), where the positive collinear phase mismatch $\Delta_{0} l_{c}$ is so large that the turning point frequency of the phase-matching curve $\Omega_{\mathrm{tp}}=\Omega^{(L)}(\bar{q}=0)=$ $-\Omega_{0} D_{0}$ falls outside the selected frequency band [i.e., we have $\left.\Omega^{(L)}(\bar{q}=0)<-\frac{1}{2} \Delta \Omega_{F}\right]$. On the other hand, if $\Delta_{0} l_{c}$ is such that $-\frac{1}{2} \Delta \Omega_{F}<\Omega_{\mathrm{tp}}<\frac{1}{2} \Delta \Omega_{F}$, we have $\bar{q}_{\text {min }}=0$, this being the situation illustrated in Figs. 10(a) and 10(b). We do not consider here the case where the whole curve falls outside the selected frequency band, corresponding to very large negative values of $\Delta_{0} l_{c}$ such that $\Omega_{\mathrm{tp}}>\frac{1}{2} \Delta \Omega_{F}$.

Since the modulus of $F_{12}(\bar{q}, t)$ does not depend on $\bar{q}$ according to Eq. (B1), we can estimate the integrated coincidence rate defined in Eq. (30) simply by multiplying $\left|F_{12}(\bar{q}, t)\right|^{2}$ with the area of $R_{\vec{q}}$. According to the definitions (B2) and (B3) this area is equal to

$$
\pi\left(\bar{q}_{\max }^{2}-\bar{q}_{\min }^{2}\right)=\pi q_{0}^{2} \frac{\Delta \Omega_{F}^{\prime}}{\Omega_{0}},
$$

where $\Delta \Omega_{\mathrm{F}}^{\prime}$ denotes the temporal bandwidth of the phasematching curve selected by the temporal frequency filter,

$$
\Delta \Omega_{F}^{\prime}=\min \left\{\Delta \Omega_{F}, \frac{\Delta \Omega_{F}}{2}-\Omega_{\mathrm{tp}}\right\} .
$$

From relations (30) and (B4) we thus obtain

$$
\begin{aligned}
C(t) & =\int_{\bar{q}_{\min }}^{\bar{q}_{\max }} \frac{d \bar{q}}{(2 \pi)^{2}} \bar{q}\left|F_{12}(\bar{q}, t)\right|^{2}, \\
& =g^{2} \frac{q_{0}^{2} \Delta \Omega_{F}^{\prime} \Omega_{0}}{4 \pi} \operatorname{Rect}\left[\frac{t-\frac{1}{2} t_{\mathrm{coh}}}{t_{\mathrm{coh}}}\right],
\end{aligned}
$$

which is a box function that extends in the time interval $0<$ $t<t_{\text {coh. }}$.

While irrelevant for the estimation of $C(t)$, the phase factor $e^{-i \Omega^{(L)}(\bar{q}) t}$ in Eq. (B1) plays an important role in the estimation of the spatially resolved coincidence rate $C_{x=0}(t)$, which, according to Eq. (32) takes the form of a coherent superposition of the amplitude function $F_{12}(\vec{q}, t)$ [see discussion after Eq. (32)]. Using again the definition of $\bar{q}_{\min }$ and $\bar{q}_{\max }$ together with the explicit expression of $\Omega^{(L)}(\bar{q})$ given in Eq. (29), we find for $\Omega_{\mathrm{tp}}<\frac{\Delta \Omega_{F}}{2}$,

$$
\begin{aligned}
\int_{R_{\vec{q}}} d \vec{q} e^{-i \Omega^{(L)}(\bar{q}) t} & =2 \pi \int_{\bar{q}_{\min }}^{\bar{q}_{\max }} d \bar{q} \bar{q} e^{-i \Omega^{(L)}(\bar{q}) t} \\
& =\pi q_{0}^{2} \frac{\Delta \Omega_{F}^{\prime}}{\Omega_{0}} e^{-i \frac{1}{2} \max \left\{\frac{\Delta \Omega_{F}}{2}+\Omega_{\mathrm{tp}}, 0\right\} t} \operatorname{sinc} \frac{\Delta \Omega_{F}^{\prime} t}{2} .
\end{aligned}
$$

Using this result, we obtain by substituting Eq. (B1) into Eq. (32),

$$
C_{x=0}(t)=g^{2}\left(\frac{\Delta \Omega_{F}^{\prime} q_{0}^{2}}{4 \pi}\right)^{2} \operatorname{Rect}\left[\frac{t-\frac{1}{2} t_{\mathrm{coh}}}{t_{\mathrm{coh}}}\right] \operatorname{sinc}^{2} \frac{\Delta \Omega_{F}^{\prime} t}{2},
$$

which, in contrast to $C(t)$, has a temporal width determined by the full PDC bandwidth intercepted in the detection process, $\Delta \Omega_{F}^{\prime}$, rather than the characteristic GVM bandwidth $\Omega_{0}$ (as long as we are assuming that $\Delta \Omega_{F}^{\prime} \gg \Omega_{0}$ ).

The found expressions of $C(t)$ and $C_{x=0}(t)$ are compared with the corresponding numerical evaluations in Fig. 5(b) and in Fig. 4(a), respectively. The smoother behavior of the numerical results (solid lines) with respect to the analytical functions (dashed lines) is due to GVD effects, that are neglected in the linearized phase-mismatch function (28). However, the overall behavior, in particular the temporal localization of both correlation functions, is well described by the previously discussed analytical estimates.
[1] A. Gatti, E. Brambilla, and Lugiato, Quantum Imaging (Elsevier, North-Holland, 2008).

[2] V. Giovannetti, S. Lloyd, and L. Maccone, Nature 412, 417 (2001).

[3] A. Valencia, G. Scarcelli, and Y. H. Shih, Appl. Phys. Lett. 85, 2655 (2004).

[4] A. F. Abouraddy, M. B. Nasr, B. E. A. Saleh, A. V. Sergienko, and M. C. Teich, Phys. Rev. A 65, 053817 (2002).

[5] M. B. Nasr, B. E. A. Saleh, A. V. Sergienko, and M. C. Teich, Opt. Express 12, 1353 (2004).

[6] S. Carrasco, J. P. Torres, L. Torner, A. Sergienko, B. E. A. Saleh, and M. C. Teich, Opt. Lett. 29, 2429 (2004).
[7] B. Dayan, A. Pe'er, A. A. Friesem, and Y. Silberberg, Phys. Rev. Lett. 93, 023005 (2004).

[8] S. E. Harris, Phys. Rev. Lett. 98, 063602 (2007).

[9] M. B. Nasr, S. Carrasco, B. E. A. Saleh, A. V. Sergienko, M. C. Teich, J. P. Torres, L. Torner, D. S. Hum, and M. M. Fejer, Phys. Rev. Lett. 100, 183601 (2008).

[10] M. Corona and A. B. U'Ren, Phys. Rev. A 76, 043829 (2007).

[11] M. Hendrych, X. Shi, A. Valencia, and J. P. Torres, Phys. Rev. A 79, 023817 (2009).

[12] A. Gatti, E. Brambilla, L. Caspani, O. Jedrkiewicz, and L. A. Lugiato, Phys. Rev. Lett. 102, 223601 (2009). 
[13] C. Conti and S. Trillo, Localized Waves (Wiley Interscience, Malden, 2007).

[14] L. Caspani, E. Brambilla, and A. Gatti, Phys. Rev. A 81, 033808 (2010).

[15] A. Joobeur, B. E. A. Saleh, and M. C. Teich, Phys. Rev. A 50, 3349 (1994)

[16] A. Gatti, R. Zambrini, M. San Miguel, and L. A. Lugiato, Phys. Rev. A 68, 053807 (2003).

[17] E. Brambilla, A. Gatti, M. Bache, and L. A. Lugiato, Phys. Rev. A 69, 023802 (2004).

[18] N. Boeuf, D. Branning, I. Chaperot, E. Dauler, S. Guerin, G. Jaeger, A. Muller, and A. Migdall, Opt. Eng. 39, 1016 (2000).

[19] L. Mandel and E. Wolf, Optical Coherence and Quantum Optics (Cambridge University Press, Cambridge, 1995).

[20] O. Jedrkiewicz, A. Picozzi, M. Clerici, D. Faccio, and P. Di Trapani, Phys. Rev. Lett. 97, 243903 (2006).
[21] O. Jedrkiewicz, M. Clerici, A. Picozzi, D. Faccio, and P. Di Trapani, Phys. Rev. A 76, 033823 (2007).

[22] L. Yu, M. C. Huang, M. Z. Chen, W. Z. Chen, W. D. Huang, and Z. Z. Zhu, Opt. Lett. 23, 409 (1998).

[23] L. R. Rabiner, R. W. Schafer, and C. M. Rader, IEEE Trans. Audio Electroacoust. 17, 86 (1969).

[24] A. Gatti, E. Brambilla, L. Caspani, and L. Lugiato (in preparation)

[25] C. K. Hong, Z. Y. Ou, and L. Mandel, Phys. Rev. Lett. 59, 2044 (1987).

[26] E. Brambilla, L. Caspani, L. Lugiato, and A. A. Gatti (in preparation).

[27] V. G. Dmitriev, G. G. Gurzadyan, and D. N. Nikogosyan, Handbook of Nonlinear Optical Crystals (Springer, Berlin, 1991). 